\title{
Organotypic Coculture Preparation for the Study of Developmental Synapse Elimination in Mammalian Brain
}

\author{
Naofumi Uesaka, ${ }^{1}$ Takayasu Mikuni, ${ }^{1}$ Kouichi Hashimoto, ${ }^{1,4,5}$ Hirokazu Hirai, ${ }^{2}$ Kenji Sakimura, ${ }^{3}$ and Masanobu Kano ${ }^{1}$ \\ ${ }^{1}$ Department of Neurophysiology, Graduate School of Medicine, The University of Tokyo, Tokyo 113-0033, Japan, ${ }^{2}$ Department of Neurophysiology, Gunma \\ University Graduate School of Medicine, Maebashi, Gunma 371-8511, Japan, ${ }^{3}$ Department of Cellular Neurobiology, Brain Research Institute, Niigata \\ University, Niigata 951-8585, Japan, ${ }^{4}$ Department of Neurophysiology, Graduate School of Biomedical Sciences, Hiroshima University, Hiroshima

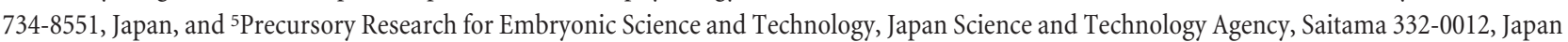

We developed an organotypic coculture preparation allowing fast and efficient identification of molecules that regulate developmental synapse elimination in the mammalian brain. This coculture consists of a cerebellar slice obtained from rat or mouse at postnatal day 9 (P9) or P10 and a medullary explant containing the inferior olive dissected from rat at embryonic day 15 . We verified that climbing fibers (CFs), the axons of inferior olivary neurons, formed functional synapses onto Purkinje cells (PCs) in the cerebellum of cocultures. PCs were initially reinnervated by multiple CFs with similar strengths. Surplus CFs were eliminated subsequently, and the remaining CFs became stronger. These changes are similar to those occurring in developing cerebellum in vivo. Importantly, the changes in $\mathrm{CF}$ innervations in cocultures involved the same molecules required for CF synapse elimination in vivo, including NMDA receptor, type 1 metabotropic glutamate receptor and glutamate receptor $\delta 2$ (GluR $\delta 2$ ). We demonstrate that gain- and loss-of-function analyses can be efficiently performed by lentiviral-mediated overexpression and RNAi-induced knockdown of GluR $\delta 2$. Using this approach, we identified neuroligin- 2 as a novel molecule that promotes CF synapse elimination in postsynaptic PCs. Thus, our coculture preparation will greatly facilitate the elucidation of molecular mechanisms of synapse elimination.

\section{Introduction}

In developing brain, neuronal connections are initially redundant, but they are refined and become functionally mature through activity-dependent competition among surplus synaptic inputs to each postsynaptic cell. Understanding how necessary synapses are selectively strengthened and stabilized while redundant connections are weakened and eventually eliminated is one of the most important challenges in neuroscience. Addressing this issue requires selection of proper model systems in the brain that allow quantitative assessment of the strength and number of synaptic inputs. The climbing fiber (CF)-to-Purkinje cell (PC) synapse in the cerebellum has been a representative model to study postnatal refinement of neural circuits in the CNS. In neonatal rodents, PCs are innervated by multiple CFs on the soma

Received March 5, 2012; revised July 2, 2012; accepted July 8, 2012.

Author contributions: N.U., K.H., and M.K. designed research; N.U. and T.M. performed research; H.H. and K.S. contributed unpublished reagents/analytic tools; N.U. and T.M. analyzed data; N.U., K.H., and M.K. wrote the paper.

This work was supported by Grants-in-Aid for Scientific Research 22300125 (K.H.), 21300118 (K.S.), and 17023021, 21220006, and 23650204 (M.K.); the Funding Program for Next Generation World-Leading Researchers (LSO21; H.H.); the Strategic Research Program for Brain Sciences (Development of Biomarker Candidates for Social Behavior); and the Global COE (Center of Excellence) Program (Integrative Life Science Based on the Study of Biosignaling Mechanisms) from the Ministry of Education, Culture, Sports, Science and Technology (MEXT), Japan. N.U. was a research fellow of the Japan Society for the Promotion of Science. We thank K. Matsuyama, M. Sekiguchi, S. Tanaka, and A. Koseki for technical assistance. We thank Dr. Arthur Nienhuis and St. Jude Children's Research Hospital and George Washington University for the lentiviral backbone vector and the packaging plasmid, Dr. Didier Trono for the psPAX2 plasmid, pCMV $\triangle$ R8.74, pMD.2G, and Dr. Peter Scheiffele for the neuroligin-2 plasmid.

Correspondence should be addressed to Masanobu Kano, Department of Neurophysiology, Graduate School of Medicine, The University of Tokyo, 7-3-1 Hongo, Tokyo 113-0033, Japan. E-mail: mkano-tky@m.u-tokyo.ac.jp.

DOI:10.1523/JNEUROSCI.1097-12.2012

Copyright $\odot 2012$ the authors $\quad 0270-6474 / 12 / 3211657-14 \$ 15.00 / 0$ with similar synaptic strengths. Subsequently, a single CF is selectively strengthened in each PC, and then surplus weaker CFs are eliminated, and most PCs become innervated by single CFs by the end of the third postnatal week (Crepel, 1982; Lohof et al., 1996; Hashimoto and Kano, 2003, 2005; Bosman et al., 2008; Kano and Hashimoto, 2009). Previous studies have identified several key molecules that are involved in CF synapse elimination by using gene knock-out (KO) mice and pharmacological manipulations (Kano and Hashimoto, 2009; Watanabe and Kano, 2011). However, generating knock-out mice is time consuming, and it is difficult to delete multiple genes in the same mouse. Pharmacological manipulation of molecules in the brain in vivo accompanies the problem of drug specificity and the difficulty in persistent, local, and stable application of drugs to the brain. Moreover, these in vivo studies are not suitable for a large-scale screening of candidate molecules that may be involved in $\mathrm{CF}$ synapse elimination. For accelerating the study of CF synapse elimination, one requires an experimental system that enables high throughput screening of candidate molecules.

Here we report an organotypic coculture consisting of a slice of the cerebellum and an explant of the medulla oblongata including the inferior olive, the origin of CFs. Importantly, CFs originating from the medullary explant formed functional synapses onto PCs with morphological and electrophysiological properties similar to those seen in vivo. We demonstrate that selective strengthening of a single CF and elimination of surplus CFs occurs in each PC of cocultures in essentially the same manners as in vivo. We also verified that pharmacological blockade, overexpression or RNAi-mediated knockdown of key molecules 
for CF synapse elimination in vivo promoted or impaired CF synapse elimination in cocultures. These results clearly demonstrate that gain-of-function and loss-of-function analyses can be easily and efficiently performed in cocultures. Interestingly, using this approach, we show a strong evidence that neuroligin-2 in postsynaptic PCs is required for CF synapse elimination. Thus, the coculture developed here is useful for high throughput screening of candidate molecules that are involved in synapse elimination in developing brain.

\section{Materials and Methods}

Animals. Sprague Dawley rats and C57BL/6 mice were used (CLEA Japan and Japan SLC). The day on which the vaginal plug was detected in the morning was designated as embryonic day 0 (E0). The first $24 \mathrm{~h}$ after birth was referred to as postnatal day $0(\mathrm{P} 0)$. All experiments were performed according to the guidelines laid down by the animal welfare committees of the University of Tokyo and the Japan Neuroscience Society. Glutamate receptor $\delta 2($ GluR $\delta 2)$ knock-out mice were obtained from the Grid2-Cre mutant mouse whose GluR $\delta 2$ gene (Grid2) has been replaced with the Cre recombinase gene by homologous recombination (Yamasaki et al., 2011). Heterozygous mutant pairs ( $\mathrm{Grid}_{2} \mathrm{Cre} / \mathrm{+}^{+}$) were mated to obtain the homozygous mutant mice (Grid2 ${ }^{\mathrm{Cre} / \mathrm{Cre}}$ ) that were used as GluR $\delta 2$ knock-out mice.

Organotypic slice culture. The methods for coculture of the cerebellum and the medulla oblongata were similar to those described previously for thalamocortical cocultures and hippocampal cultures (Yamamoto et al., 1989; Stoppini et al., 1991). We tested various parameters, including the age of the animal, method for preparing slices, membrane filter for placing slices and medullary explants, material for coating the membrane, composition of the culture medium, and volume and frequency of culture medium change. We achieved the best result when cerebellar slices and medullary explants were prepared in the following condition (Fig. 1). E15 rat embryos were obtained by cesarean delivery from pregnant females anesthetized with isoflurane or pentobarbital. The whole brain was dissected after decapitation from each fetal rat and kept in cold HBSS. Then, the ventral medial portion of the medulla containing inferior olivary neurons was dissected. The cerebellum was dissected out from rats or mice at P9 or P10 that were deeply anesthetized with isoflurane under a dissecting microscope. Cerebellar slices with $300 \mu \mathrm{m}$ (for rats) or 250 $\mu \mathrm{m}$ (for mice) thickness were cut from the vermis using a slicer (LinearSlicer Pro 7; Dosaka). A block of the medulla was plated at the ventricular side of the cerebellar slice on a membrane filter (Millicell-CM PICMORG50; Millipore), which was coated with rat-tail collagen. The culture medium consisted of 50\% MEM, 25\% horse serum (Invitrogen or STEMCELL Technologies), 25\% HBSS, 3 mm GlutaMAX (Invitrogen), $10 \mu \mathrm{M}$ Mifepristone (Ru486; Tocris Bioscience), and $5 \mathrm{mg} / \mathrm{ml}$ glucose. For cocultures of the mouse cerebellum with the rat medulla oblongata, the culture medium contained $2.5 \%$ HBSS and $22.5 \%$ distilled water with $2 \%$ B27 supplements instead of $25 \%$ HBSS. These cultures were maintained at $37^{\circ} \mathrm{C}$ in an environment of humidified $95 \%$ air and $5 \% \mathrm{CO}_{2}$. One-half of the culture medium was exchanged with fresh medium every other day. The level of the medium was adjusted slightly below the surface of the explants so that they could receive a sufficient supply of the culture medium and the mixed gas. This procedure was crucial for the survival of cerebellar and medullary cells in the explants.

Histology. For immunohistochemistry, cerebellar slices were fixed in $4 \%$ paraformaldehyde in $0.1 \mathrm{~m}$ phosphate buffer, $\mathrm{pH} 7.4$, for $30 \mathrm{~min}$ at
C

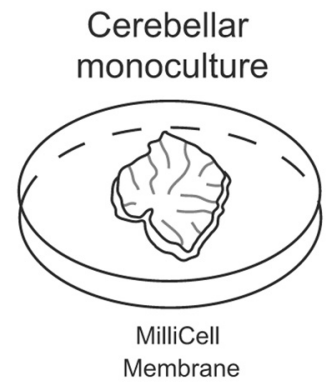

Olivo-cerebellar coculture

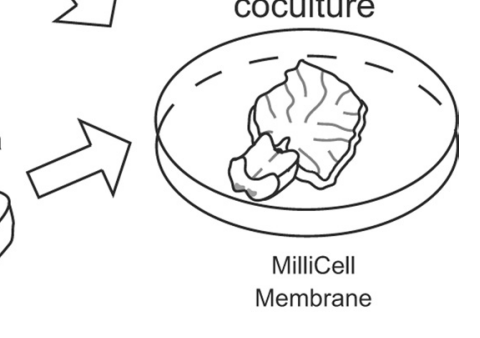

olive
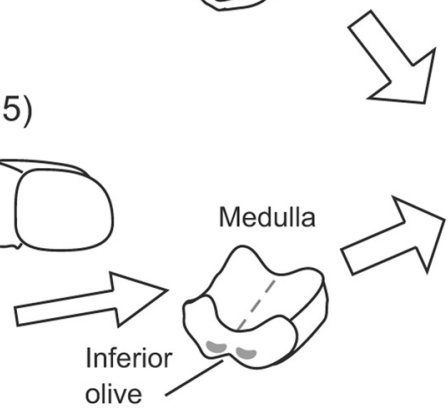

Membran

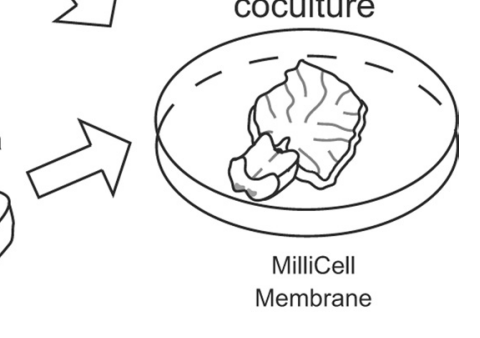

Figure 1. Schematic diagram of the method for preparing cocultures of the cerebellum and the medulla. $A$, The cerebellum was dissected out from rats or mice at P9 or P10 under deep anesthesia. Cerebellar slices with $300 \mu \mathrm{m}$ (for rats) or $250 \mu \mathrm{m}$ (for mice) thickness were dissected from the vermis. Scissors represent the sites of sectioning. $\boldsymbol{B}$, The whole brain was dissected after decapitation from each fetal rat at $\mathrm{E} 15$, and the medulla containing inferior olivary neurons was dissected. $C$, A block of the medulla was plated at the ventricular side of a cerebellar slice on a membrane filter that was coated with rat-tail collagen.

room temperature. After washing in PBS, the slices were incubated for $2 \mathrm{~h}$ in PBS containing $0.5 \%$ Triton X-100 and $10 \%$ donkey serum. For immunocytochemistry, HEK293T cells were fixed one day after the transfection with $4 \%$ paraformaldehyde in $0.1 \mathrm{M}$ phosphate buffer for $10 \mathrm{~min}$ followed by permeabilization with $0.05 \%$ Triton X-100 for $30 \mathrm{~min}$. Then the mouse monoclonal antibody $(\mathrm{Ab})$ against calbindin $\mathrm{D}-28 \mathrm{~K}(1: 3000$; Sigma) to visualize PCs, the $\mathrm{Ab}$ against guinea pig vesicular glutamate transporter 1 (VGluT1; 1:1000; Millipore Bioscience Research Reagents) to label PF terminals, the Ab against guinea pig VGluT2; 1:1000; Millipore Bioscience Research Reagents) to label CF terminals, the Ab against rabbit GluR $\delta 2$ (1:200; Frontier Institute), the $A b$ against rabbit neuroligin-2 (1:500; Synaptic Systems), the Ab against guinea pig glutamate-aspartate transporter (GLAST) (1:200; Frontier Institute) to label Bergmann glia, the Ab against rabbit parvalbumin (1:200; Frontier Institute) to label inhibitory neurons, or the $\mathrm{Ab}$ against green fluorescent protein (GFP; 1:1000; Nacalai Tesque) were applied overnight at $4^{\circ} \mathrm{C}$. After $4 \mathrm{~h}$ incubation at room temperature with secondary antibodies against donkey anti-mouse Alexa Fluor 488 (1:400; Invitrogen), donkey anti-guinea pig CY3 (1:300; Jackson ImmunoResearch), donkey antirabbit Alexa Fluor 488 (1:400; Invitrogen), donkey anti-rabbit CY3, or donkey anti-rat Alexa Fluor 488 (1:400; Invitrogen), the slices were washed several times in PBS. The immunolabeled sections and dishes were examined with a confocal laser scanning microscope (Zeiss LSM 510).

Laminar configuration in cocultures was determined by Nissl staining. Cocultures were fixed by immersion in $4 \%$ paraformaldehyde in $0.1 \mathrm{M}$ phosphate buffer and kept in 30\% sucrose in PBS. They were embedded on an agar block (4\% agar in PBS), cut into $20 \mu \mathrm{m}$ frozen sections, and stained with cresyl violet or hematoxylin and eosin.

Electrophysiology. For stable recording of synaptic responses, most part of the medullary explant was cut from the coculture with a knife, and CFs in the remaining portion of the explants were isolated from the somata of neurons in the inferior olive. This procedure reduced synaptic responses in PCs elicited by spontaneous firing of inferior olivary neurons. The cocultures were recovered for at least $30 \mathrm{~min}$ at room temperature in a reservoir chamber bathed with a solution containing the following (in mM): $125 \mathrm{NaCl}, 2.5 \mathrm{KCl}, 2 \mathrm{CaCl}_{2}, 1 \mathrm{MgSO}_{4}, 1.25 \mathrm{NaH}_{2} \mathrm{PO}_{4}, 26 \mathrm{NaHCO}_{3}$, and 21 glucose bubbled with $95 \% \mathrm{O}_{2}$ and $5 \% \mathrm{CO}_{2}$. Cocultures were transferred to a recording chamber on the stage of an Olympus BX51WI 


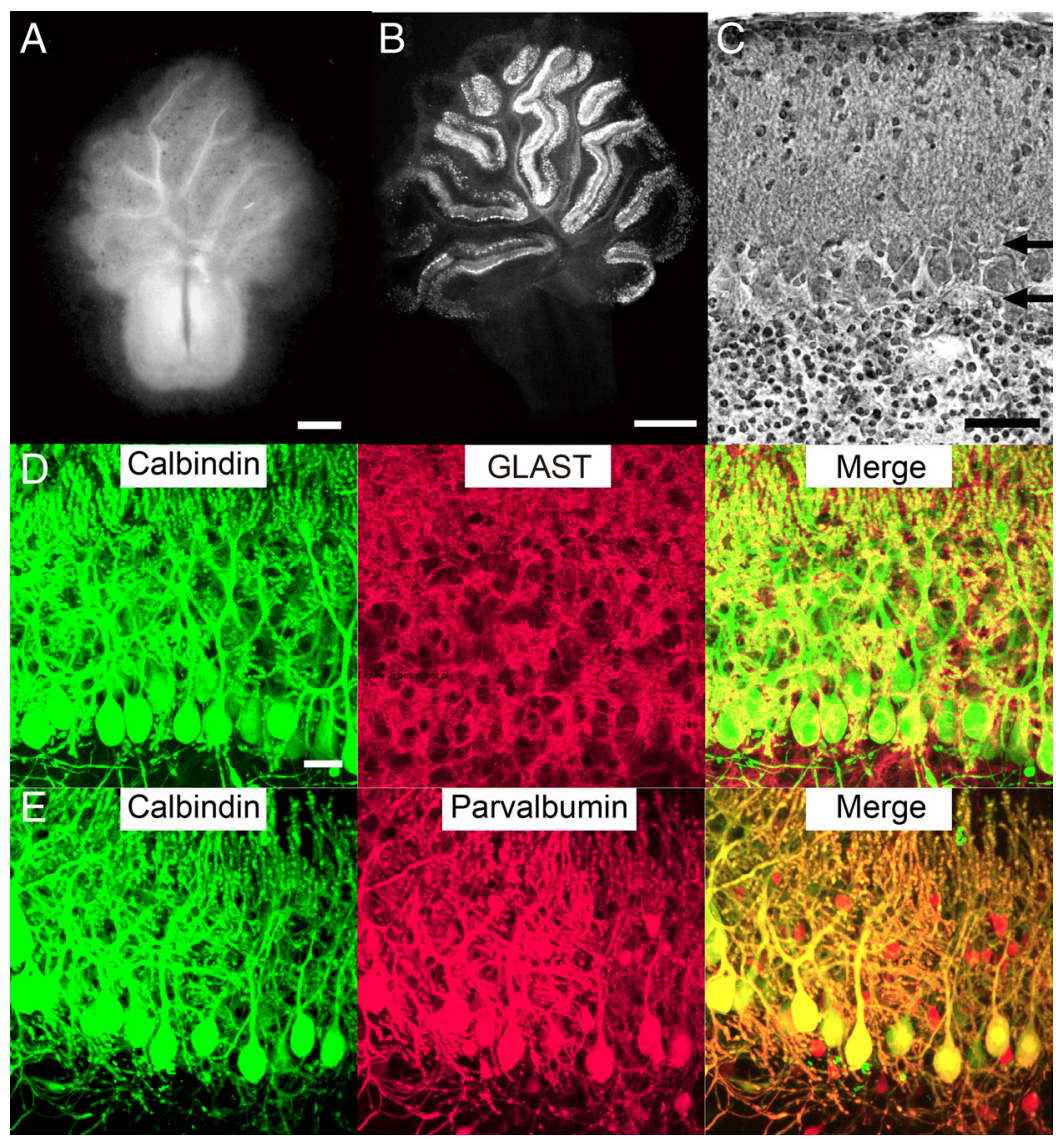

Figure 2. Cytoarchitecture in cocultures. A, Dark-field micrograph of a coculture at 3 weeks in vitro. B, Fluorescent immunohistochemistry using an antibody against the $\mathrm{PC}$ marker calbindin D-28K at 1 week in vitro. C, Niss--stained section of the cultured cerebellar slice at 1 week in vitro. Horizontal arrows show the boundaries between the $\mathrm{PC}$ layer and the molecular layer (top arrow) or the granule cell layer (bottom arrow). D, E, Bergmann glia and inhibitory interneurons in cocultures. After 10 DIV, cocultures were double immunostained for calbindin (green) and GLAST, a marker of Bergmann glia (D; red), or for calbindin (green) and parvalbumin, a marker of inhibitory neurons ( $\boldsymbol{E}$; red). Note that GLAST signals are closely associated with calbindin signals and that calbindin-negative/parvalbumin-positive inhibitory neurons are present in the molecular layer and the $P C$ layer. Scale bars: $A, 0.5$ $\mathrm{mm} ; \boldsymbol{B}, 1 \mathrm{~mm} ; \boldsymbol{C}, 50 \mu \mathrm{m} ; \boldsymbol{D}$ (for $\boldsymbol{D}, \boldsymbol{E}), 20 \mu \mathrm{m}$.

microscope (Olympus Optical). The recording chamber was continuously perfused with oxygenated bath solution supplemented with bicuculline $(10 \mu \mathrm{M})$ or picrotoxin $(100 \mu \mathrm{M})$ to block inhibitory synaptic transmission. 3- $((R)$-2-carboxypiperazine-4-yl)-propyl-1-phosphonic acid (R-CPP; $5 \mu \mathrm{M})$ was also added to suppress polysynaptic responses and inhibit spontaneous EPSC when the number of CF-EPSC steps was examined. Whole-cell recordings were made from visually identified or GFP-positive PCs using an upright and a fluorescence microscope at room temperature $\left(24^{\circ} \mathrm{C}\right.$; for Figs. 5, 6, 7, and 9) or $32^{\circ} \mathrm{C}$ (for all other figures). For stable recording of CF-EPSCs under voltage-clamp mode (see Figs. 5A, C, 6, 7, 9-14), we used the following intracellular solution (solution 1), which was designed to minimize the fluctuation of membrane voltage of PCs at holding potential of -10 or $-20 \mathrm{mV}$ (Kano et al., 1995, 1997; Hashimoto and Kano, 2003; Hashimoto et al., 2009a). The composition of solution 1 was as follows (in $\mathrm{mm}$ ): $60 \mathrm{CsCl}, 10 \mathrm{Cs}$ D-gluconate, 20 tetraethylammonium-Cl, 20 BAPTA, $4 \mathrm{MgCl}_{2}$, 4 ATP, 0.4 GTP, and 30 HEPES, pH7.3, adjusted with $\mathrm{CsOH}$ for EPSC. For recording CF- or PF-mediated EPSPs under current-clamp mode (Fig. $5 B, D$ ), an intracellular solution (solution 2) with the following composition (in mM) was used: $130 \mathrm{~K}$ D-gluconate, $6 \mathrm{KCl}, 10 \mathrm{NaCl}, 10 \mathrm{HEPES}$, $0.16 \mathrm{CaCl}_{2}, 2 \mathrm{MgCl}_{2}, 0.5$ EGTA, $4 \mathrm{Na}-\mathrm{ATP}$, and $0.4 \mathrm{Na}-\mathrm{GTP}, \mathrm{pH}$ 7.3, adjusted with $\mathrm{KOH}$. The resistance of patch pipettes was $2-4 \mathrm{M} \Omega$ when filled with either of the two solutions. The pipette access resistance was compensated by $70 \%$. Signals were low-pass filtered at $0.05-2 \mathrm{kHz}$ and sampled at $0.1-10 \mathrm{kHz}$ using a voltage-clamp amplifier (EPC 10; HEKA). Online data acquisition and off-line data analysis were performed using
PULSE software (HEKA). The holding potential was corrected for liquid junction potential. To activate CFs, four tungsten electrodes (3.5$4.5 \mathrm{M} \Omega$; catalog \#UEWMGCSEKNNM; FHC) were placed in the remaining portions of medullary explants. Electrical pulses (duration, 0.1 or $0.5 \mathrm{~ms}$; amplitude, $0-90 \mathrm{~V}$ ) were applied between all the possible combination of two electrodes, and the number of discrete CFEPSCs elicited in each PC was examined.

Chronic drug treatments. To block glutamate receptors, culture media containing the following drugs were applied from $7 \mathrm{~d}$ in vitro (DIV) to the day of recording: $R-C P P(10 \mu \mathrm{M})$ and $(S)-(+)-\alpha-$ amino-4-carboxy-2-methylbenzeneacetic acid (LY367385; $100 \mu \mathrm{m}$; Tocris Bioscience).

Preparation of viral vector constructs. Vesicular stomatitis virus $\mathrm{G}$ (VSV-G) pseudotyped lentiviral vectors provided by St. Jude Children's Research Hospital (pCL20c) (Hanawa et al., 2002) were used in this study. The vectors were designed to express GFP, GluR $\delta 2$, and/or microRNA ( $\mathrm{miR}$ ) directed against GluR $\delta 2$ or neuroligin-2 under the control of a truncated L7 promoter (pCL20c-trL7) (Sawada et al., 2010). The GluR $\delta 2$ and microRNA constructs were produced by PCR followed by subcloning into the pCL20c-trL7 vector. Full-length cDNA of mouse GluR $\delta 2$ was cloned by PCR with primers ( $5^{\prime}$-GA TCGGGAATTCATGGAAGTTTTCCCCTTGC TC- $3^{\prime}$ and 5'-ATACGGCGCGGCCGCTCATA TGGACGTGCCTCGGTCGGG-3'). The plasmid with mouse neuroligin-2 construct was from Addgene (plasmid 15246) (Chih et al., 2006). An RNAi-resistant mutation of neuroligin-2 cDNA (neuroligin-2 RES) was introduced using the QuikChange Site-Directed Mutagenesis Kit (Agilent Technologies; primer, GTGGTAGCGGGC AGGCTGCACGTCTTGATCGACCAGTTCCC GGGAAGACTTCCGG). mOrange2 (Clontech) and neuroligin-2 RES were linked in-frame, interposed by picornavirus "self-cleaving" P2A peptide sequence to enable efficient bicistronic expression, and then subcloned into pCL20ctrL7. We validated these constructs in HEK293T cells and cerebellar slices by immunocytochemistry and immunohistochemistry (Fig. 14). For vectorbased RNAi analysis of GluR $\delta 2$ and neuroligin-2, we used the BLOCK-iT Pol II miR RNAi Expression Vector Kit (Invitrogen). Engineered microRNAs (5'-TGCTGTATGCATGGCATCTGCCAAAGGTTTTGGCCACTGACTG ACCTTTGGCATGCCATGCATA- ${ }^{\prime}$ ' and 5' $^{\prime}$-CCTGTATGCATGGCATGC CAAAGGTCAGTCAGTGGCCAAAACCTTTGGCAGATGCCATGCATA C-3' for GluR 82 -microRNA; 5'-TGCTGTGTACATCCTGGTCCACTAG CGTTTTGGCCACTGACTGACGCTAGTGGCAGGATGTACA-3' and 5' - CCTGTGTACATCCTGCCACTAGCGTCAGTCAGTGGCCAAAAC GCTAGTGGACCAGGATGTACAC-3' for neuroligin-2-microRNA) were designed against the mouse GluR $\delta 2$ - or neuroligin-2-coding sequence following the BLOCK-iT Pol II miR RNAi Expression Vector Kit guidelines (Invitrogen). These oligonucleotides were subcloned into a pCL20c-trL7 vector.

Virus preparation and infection. The virus vector was produced by cotransfection of HEK293T cells $\left(2-6 \times 10^{6}\right.$ per dish) with a mixture of two packaging plasmids [ $8 \mu \mathrm{g}$ of pCMV $\Delta \mathrm{R} 8.74$ and $3 \mu \mathrm{g}$ of pMD.2G (generous gifts from Karl Deisseroth, Howard Hughes Medical Institute, Stanford University, Stanford, CA) or $7 \mu \mathrm{g}$ of psPAX2 (Addgene; plasmid 15246) and $3 \mu \mathrm{g}$ of pCAG-VSV-G (a generous gift from Arthur Nienhuis, St. Jude Children's Research Hospital, Memphis, TN) (Niwa et al., 1991)] and one lentivirus vector $(10 \mu \mathrm{g}$ of vector plasmid pCL20ctrL7GluR82-trL7GFP, pCL20c-trL7-GluR82miR-GFP, pCL20c-trL7neuroligin-2miR-GFP, or pCL20c-trL7-neuroligin-2-P2A-mOrange2) 
using a calcium phosphate precipitation method as reported previously (Torashima et al., 2006). Briefly, cells were cultured in DMEM (Invitrogen) supplemented with $10 \%$ fetal bovine serum (Hyclone), $100 \mathrm{U} / \mathrm{ml}$ penicillin $\mathrm{G}$, and $0.1 \mathrm{mg} / \mathrm{ml}$ streptomycin, $\mathrm{pH} 7.35$, at $37^{\circ} \mathrm{C}$ in a $5 \% \mathrm{CO}_{2}$ atmosphere. Sixteen hours after transfection, the culture medium was exchanged with fresh medium. The medium containing vector particles was harvested at $40 \mathrm{~h}$ after transfection. The medium samples were filtered through $0.22 \mu \mathrm{m}$ membranes and centrifuged at 27,000 rpm for 90 $\mathrm{min}$. The virus samples were finally suspended in $30 \mu \mathrm{l}$ of culture medium, $\mathrm{pH} 7.4$, and stored at $4^{\circ} \mathrm{C}$. PCs were infected by adding directly on the slices the volume of viral stock corresponding to $0.2-1 \times 10^{5} \mathrm{TU}(0.5 \mu \mathrm{l})$ per coculture with a microsyringe with a 33-gauge needle (Ito).

Gene delivery into medullary cells. The coding region of egfp was cloned into a pCAGGS vector (Niwa et al., 1991). Electroporation with glass microelectrodes was performed after $1 \mathrm{~d}$ in coculture to introduce the vector into medullary cells (Uesaka et al., 2007, 2008). In brief, the plasmid solution of pCAGGS-egfp $(1-2 \mu \mathrm{g} / \mu \mathrm{l})$ was pressure ejected onto the surface of the medullary explants from a glass micropipette, and electrical pulses ( 10 trains of 200 square pulses of $1 \mathrm{~ms} \mathrm{du}$ ration, $200 \mathrm{~Hz}, 500 \mu \mathrm{A}$ ) were applied through the other glass microelectrode.

Statistical analysis. A total of 170 cocultures were used for the morphological and electrophysiological experiments. All statistical values are presented as the mean \pm SEM. A MannWhitney $U$ test was used when two independent data sets were compared. For multiple comparison, data were analyzed by using the nonparametric Steel-Dwass test.

\section{Results}

\section{Cytoarchitecture and synaptic} organization of the cerebellum in cocultures

Our organotypic coculture consists of a parasagittal cerebellar slice with an explant of the medulla oblongata containing the inferior olive (Fig. 1). First, we tested various ages of animals to find out the optimal conditions for olivocerebellar cocultures. We obtained the best results when parasagittal cerebellar slices were cut from rats or mice at P9 or P10 using a vibroslicer (Fig. $1 A$ ) and a block of the medulla was dissected from rat embryos at E15 (Fig. 1B) (see Discussion). Cerebellar slices were cultured on MilliCell membrane either alone (cerebellar monoculture) (Fig. 1C) or with a medullary explant attached to the ventricular side (olivocerebellar coculture) (Fig. 1C). We found that gross anatomy, foliation, and layer structure of the cerebellum in our cocultures were similar to those of the cerebellum in vivo (Fig. 2A,B) (Altman and Bayer, 1997). Nissl staining showed that the molecular layer, the PC layer, and the granule cell layer were well preserved in cocultures (Fig. 2C) (Altman and Bayer, 1997). Many PCs appeared viable and were arranged in a row (Fig. $2 B, C$ ) and dendrites of PCs well extended in the molecular layer toward the pial surface (Fig. $2 D, E$ ). Double staining with a PC marker, calbindin, and a Bergmann glial marker, GLAST,

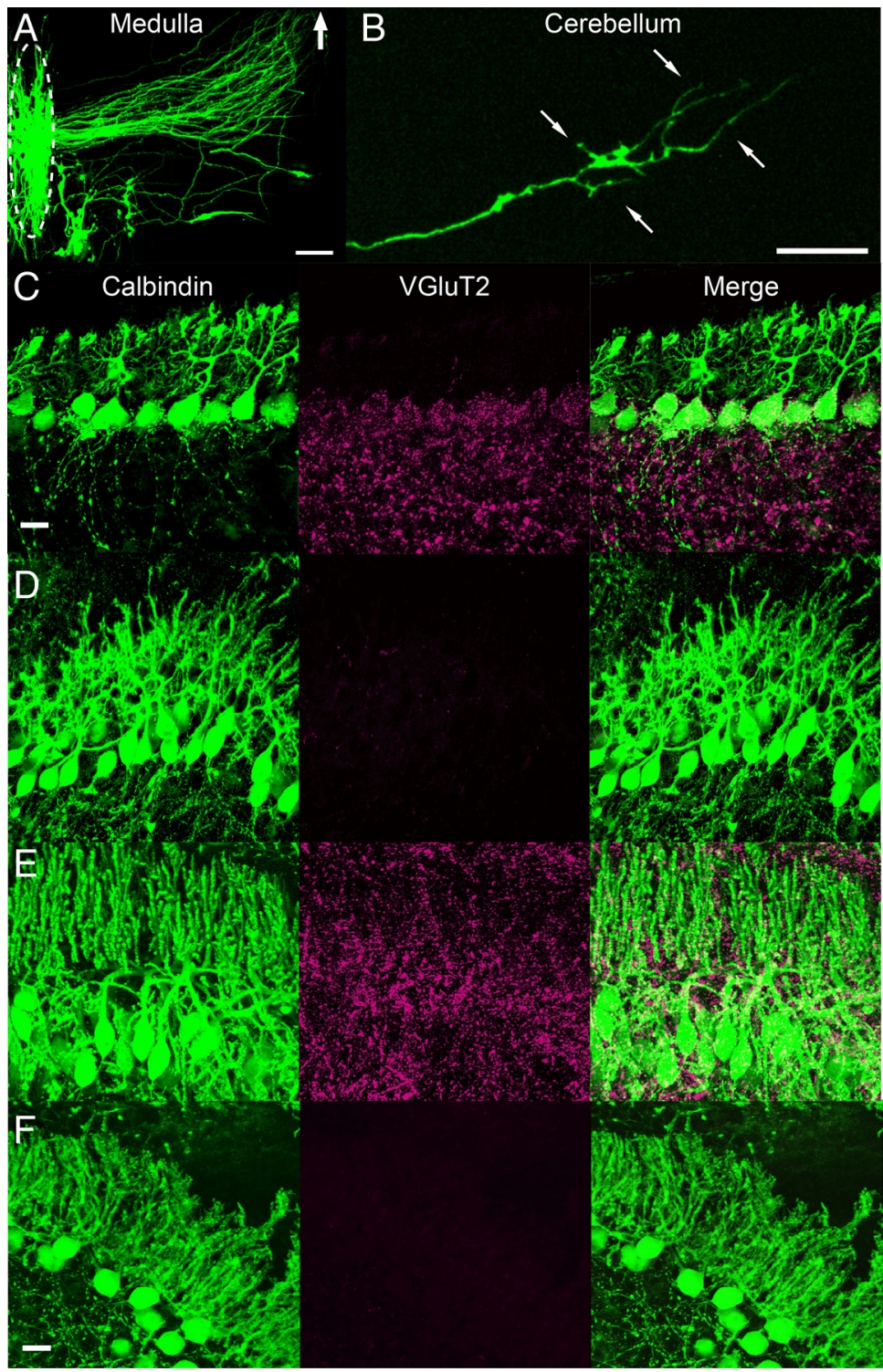

Figure 3. Synapse formation by axons from medullary explants to PCs in cocultures. $A, B$, EGFP-labeled medullary cells and their axons in cocultures after 10 DIV. Fibers from the medullary cells projected toward the cerebellar slice $(\boldsymbol{A})$ and formed terminal arbors in the $P C$ layer $(\boldsymbol{B})$. The location of cell bodies in the medulla is delineated by an oval with broken line in $\boldsymbol{A}$. The thick arrow of cocultures for calbindin (green) and a CF marker, VGluT2 (red), at $0(\boldsymbol{C}), 4(\boldsymbol{D})$ and 7 DIV (E). F, Double immunostaining of cerebellar monocultures at 7 DIV. Note that VGluT2 signals are present in PCS at 7 DIV in cocultures $(\boldsymbol{E})$ but absent in cerebellar monocultures at 7 DIV $(\boldsymbol{F})$. Scale bars: $\boldsymbol{A}, 100 \mu \mathrm{m} ; \boldsymbol{B}, \boldsymbol{C}($ for $\boldsymbol{C}-\boldsymbol{E}), \boldsymbol{F}, 20 \mu \mathrm{m}$.

demonstrated that Bergmann glia had close contacts with PC somata and dendrites at 1 week in vitro (Fig. 2D). Furthermore, parvalbumin-positive and calbindin-negative inhibitory interneurons were abundant in the molecular layer and PC layer at 1 week in vitro (Fig. $2 E$ ). These results clearly indicate that the cytoarchitecture and layer structure of the cerebellum were well preserved in cocultures.

We then examined synaptic organization of PCs in cocultures. Many axons in the medulla labeled with EGFP crossed the midline and extended toward the cerebellar slice (Fig. $3 A$ ). These axons entered into the cerebellar slice, reached the PC layer, branched off, and formed terminal arbors (Fig. 3B). This characteristic morphology was similar to that of CFs in the rat cerebellum at P4-P7 in vivo (Sugihara, 2005). We then examined CF synapses onto PCs by immunohistochemistry using an antibody 


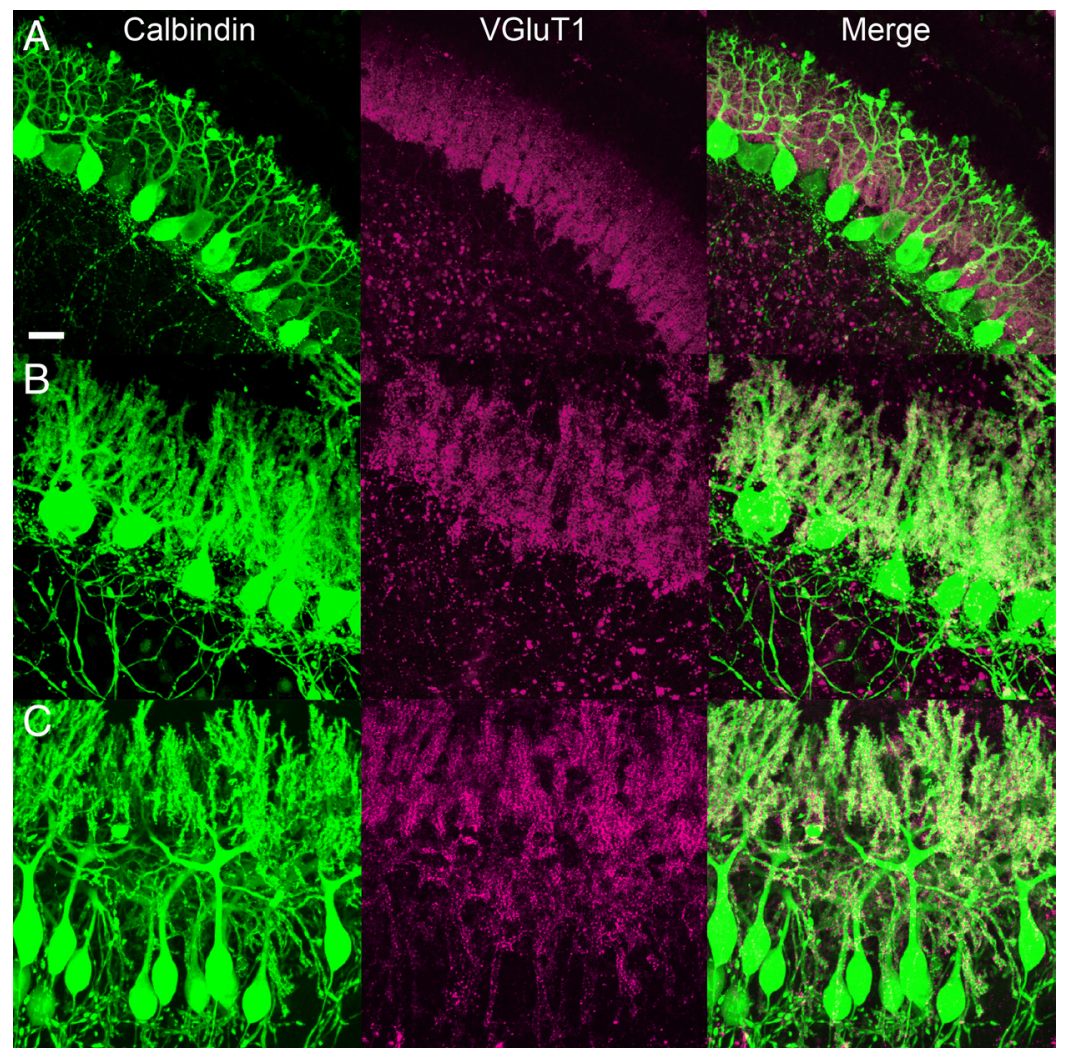

Figure 4. PF synapses on PCs in cocultures. $A-C$, Cocultures were double immunostained for calbindin (green) and a PF marker, VGluT1 (red), after $0(\boldsymbol{A}), 4(\boldsymbol{B})$, and 7 DIV (C). Note that VGluT1 signals are present in PCs throughout coculture period. Scale bar: $20 \mu \mathrm{m}$.
A

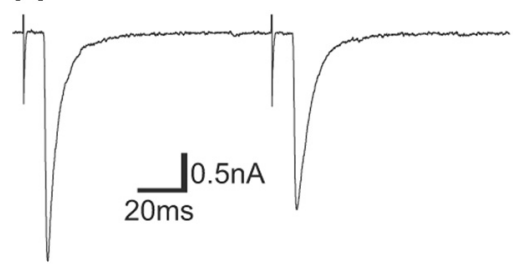

C

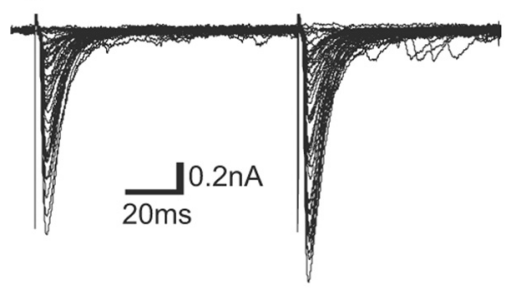

B

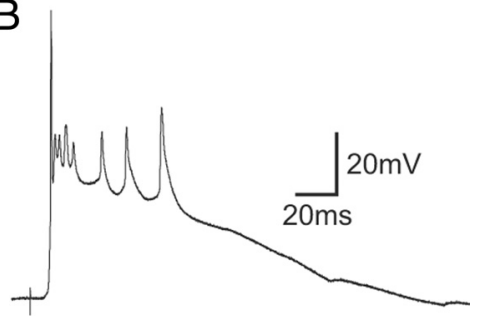

$\mathrm{D}$

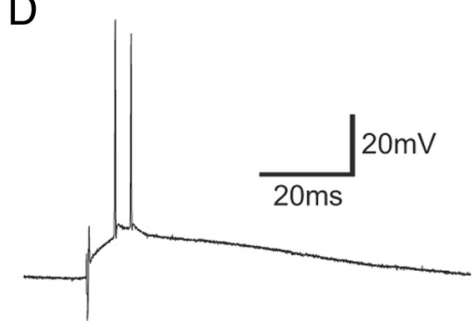

Figure 5. Electrophysiological properties of CF-PC and PF-PC synapses in cocultures. $A$, A representative trace of EPSC elicited by stimulation in the medulla under voltage-clamp mode at a holding potential of $-30 \mathrm{mV}$. A single trace recorded around threshold stimulus intensities is shown. $\boldsymbol{B}$, A representative trace of complex spike elicited by stimulation in the medulla under current-clamp mode. $\boldsymbol{C}$, Representative traces of EPSCs elicited by stimulation in the molecular layer under voltage-clamp mode at a holding potential of $-80 \mathrm{mV}$. Traces recorded while increasing the stimulus intensity were superimposed. $\boldsymbol{D}$, A representative trace of EPSP and spikes elicited by stimulation in the molecular layer 50-100 $\mu \mathrm{m}$ away from the PC soma under current-clamp mode.

against VGluT2, a specific marker for CF synaptic terminals. Consistent with the previous report for the cerebellum in vivo (Hashimoto et al., 2009a), VGluT2 signals were observed mainly on the soma of PCs at P10 when cerebellar slices were prepared (Fig. 3C). The VGluT2 signals disappeared within 4 DIV (Fig.
$3 D$ ), indicating degeneration of intrinsic CFs that had been present in cerebellar slices. Then, VGluT2 signals reappeared on PCs after 1 week in coculture (Fig. 3E). These VGluT2 signals are thought to originate from newly formed synapses between PCs and axons from the medullary explant because VGluT2 signals were absent in cerebellar monocultures without medullary explants (Fig. $3 F$ ). In contrast, VGluT1 signals were present in PC dendrites throughout culture period (Fig. $4 A-C)$.

To test whether synapses in cocultures are functional, we made whole-cell recordings from PCs. In acute cerebellar slices, responses of PCs to stimulation of PFs and CFs are known to display paired-pulse facilitation (PPF) and depression (PPD), respectively (Konnerth et al., 1990). It has also been shown that PCs exhibit two forms of action potentials, called simple and complex spikes. Simple spikes are generated depending on PCs' intrinsic membrane properties and also by phasic PF inputs, whereas complex spikes are generated exclusively by $\mathrm{CF}$ inputs. Under voltage-clamp mode, stimulation of the medullary explants elicited large EPSCs that displayed PPD (Fig. 5A). In contrast, stimulation in the molecular layer elicited EPSCs that displayed PPF and whose amplitudes were graded to stimulus strength (Fig. 5C). Under current-clamp mode, stimulation of the medullary explants elicited typical complex spikes (Fig. $5 B$ ), whereas stimulation in the molecular layer elicited simple spikes in PCs (Fig. 5D).

To obtain further evidence about the origins of excitatory inputs to PCs in cocultures, we examined the pharmacological properties of EPSCs. PCs are known to express type 1 metabotropic glutamate receptor (mGluR1), whose activation by its agonist, (S)-3,5-dihydroxyphenylglycine (DHPG), causes a reversible suppression of both CF-PC and PF-PC synaptic transmission through endocannabinoid-mediated retrograde signaling (Maejima et al., 2001, 2005). PF terminals possess type 4 metabotropic glutamate receptor and are sensitive to a group III mGluR agonist, L- (+)-2amino-4-phosphonobutyric acid (L-AP4) (Pekhletski et al., 1996), whereas CF terminals posses group II mGluRs and are sensitive to their agonist, $\left(2 S, 2^{\prime} R, 3^{\prime} R\right)$ 2 - $\left(2^{\prime}, 3^{\prime}\right.$-dicarboxycyclopropyl) glycine (DCG-IV) (Maejima et al., 2001). In cocultures, EPSCs elicited by medullary stimulation, which displayed PPD, were reversibly suppressed by DHPG $(n=8 ; 66.4 \pm 3.6 \%)$, and DCG-IV $(n=10 ; 35.8 \pm 5.3 \%)$, but not by L-AP4 $(n=5 ; 97.2 \pm 4.2 \%)$ (Fig. $6 A, B)$, when these agonists were bath applied. In contrast, EPSCs elicited by molecular layer stimulation, which showed PPF, were reversibly suppressed by DHPG 
$(n=11 ; 48.1 \pm 3.7 \%)$ and L-AP4 $(n=11$; $41.3 \pm 7.2 \%)$, but not by DCG-IV $(n=7$; $94.1 \pm 3.9 \%$ ) (Fig. $6 D, E$ ). Notably, these forms of agonist-induced suppression accompanied clear changes in paired-pulse ratio (Fig. 6C,F), indicating that the suppression was of presynaptic origin. These results collectively indicate that the stimulation in the medullary explant activated CFs that had grown into the cerebellum and innervated PCs, and the stimulation in the molecular layer activated PFs that had grown presumably from granule cells in the cerebellum. These results are consistent with the previous studies of cerebellar monocultures and olivocerebellar slice cultures (Knöpfel et al., 1990; Mariani et al., 1991; Tanaka et al., 1994).

\section{Development of CF-PC synapses}

Then, we followed developmental course of CF-PC synapses in cocultures. Consistent with the immunohistochemical data for VGluT2 (Fig. 3), CF-mediated EPSCs were not detected at $4 \mathrm{DIV}$, and they appeared at $\sim 7$ DIV (Fig. 7). We noticed that multiple CF-EPSCs could be elicited in a given $\mathrm{PC}$ when the intensity of stimulation in the medullary explant was increased gradually, indicating that most PCs were innervated by multiple CFs at 7 DIV. As shown previously in acute cerebellar slices (Kano et al., 1995, 1997; Hashimoto and Kano, 2003; Hashimoto et al., 2009a), the number of CFs innervating a given $\mathrm{PC}$ can be estimated by counting the number of discrete CF-EPSC steps elicited in that cell. We found that $~ 70 \%$ of PCs had more than four CF-EPSC steps at 7-9 DIV (Fig. 7 A,E). At 11-13 DIV, the percentage of PCs with more than four CFs decreased significantly, and the fractions of PCs innervated by one to three CFs increased (Fig. 7 B, F) $(n=54$ for $7-9$ DIV; $n=42$ for $11-13$ DIV; $p<0.001$, Mann-Whitney $U$ test). At 14-16 DIV, the percentage of PCs with more than four CFs decreased further and $\sim 40 \%$ of PCs were innervated by single CFs (Fig. 7C,G) ( $n=42$ for $11-13$ DIV; $n=98$ for $14-16$ DIV; $p<0.01$, Mann-Whitney $U$ test). However, elimination of CFs did not proceed further at 18-25 DIV (Fig. 7D,H) ( $n=98$ for $14-16$ DIV; $n=43$ for $18-25$ DIV; $p>0.1$, Mann-Whitney $U$ test). Approximately two CFs on average innervated PCs after 3 weeks in culture (Fig. $8 A$ ). These results indicate that elimination of surplus CFs occurs in cocultures as in the cerebellum in vivo, although mono CF innervation cannot be attained in $\sim 60 \%$ of PCs.

It has been shown that the EPSC amplitudes of single CFs progressively be-
A
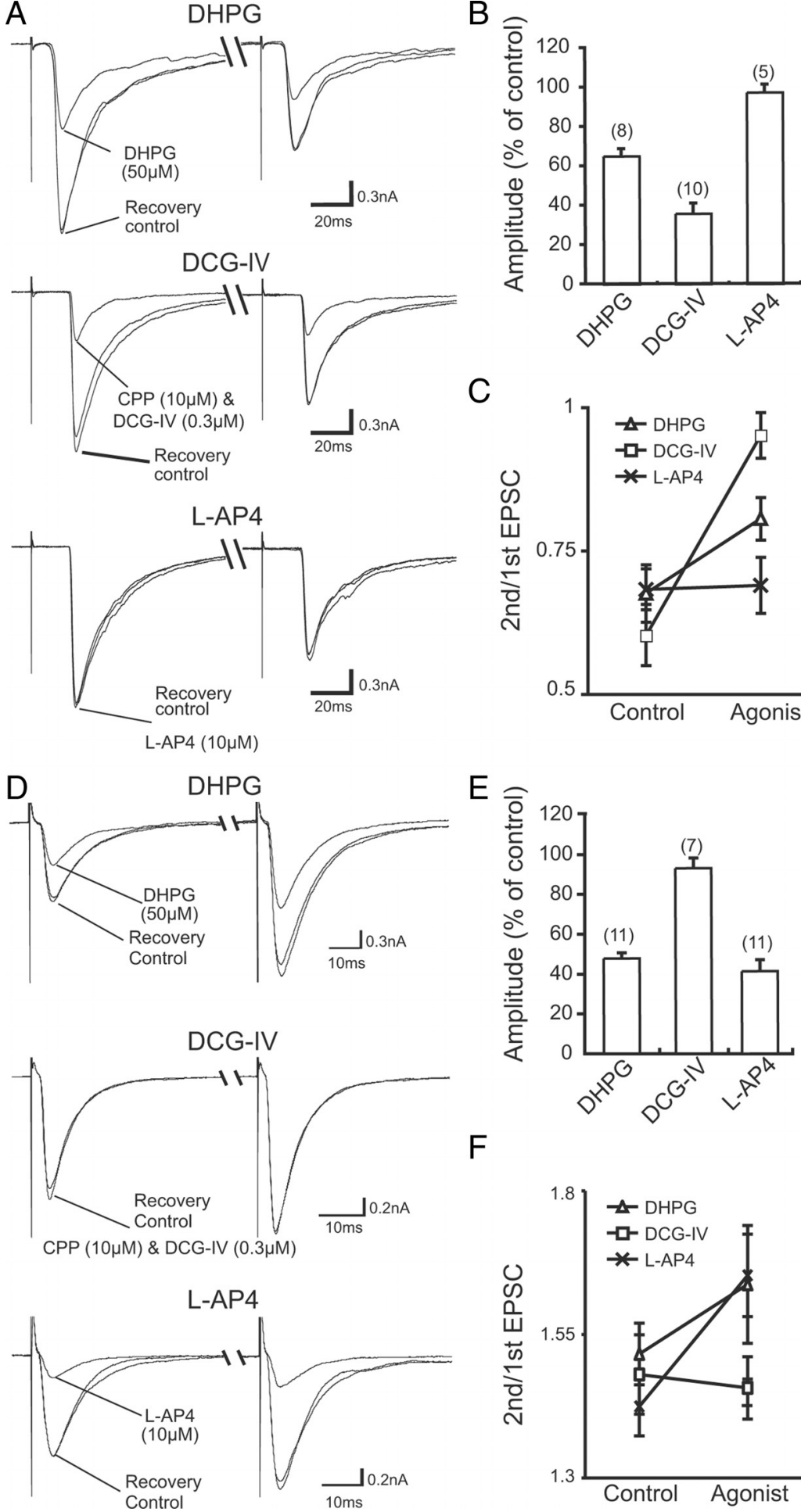

$\mathrm{F}$
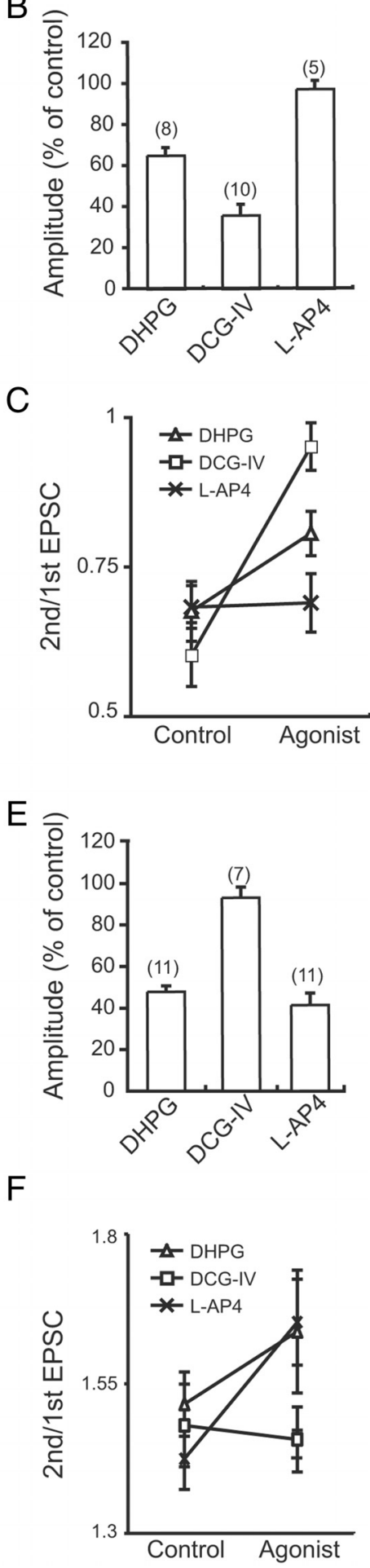

Figure 6. Pharmacological properties of CF-PC and PF-PC synapses in cocultures. A, Presynaptic inhibition of CF-EPSCs caused by $\mathrm{mGluR}$ agonists. Traces recorded before (control), during, and after (recovery) the agonist application were superimposed. The response was suppressed by $50 \mu \mathrm{M}$ DHPG (top) and $0.3 \mu \mathrm{M}$ DCG-IV (middle), but not by $10 \mu \mathrm{M} \mathrm{L-AP4}$ (bottom). The holding potential was $-30 \mathrm{mV}$. B, Summary bar graph showing the magnitude of suppression of the peak EPSC amplitudes by mGluR agonists. C, Summary of changes in paired-pulse ratio of CF-EPSCs recorded before (control) and during bath application (agonist) of DHPG, DCG-IV, and L-AP4. D, Presynaptic inhibition of PF-EPSCs caused by mGluR agonists is illustrated as in $\boldsymbol{A}$. The response was suppressed by DHPG (top) and L-AP4 (bottom), but not by DCG-IV (middle). The holding potential was $-80 \mathrm{mV}$. E, F, Summary graphs showing the magnitude of suppression of the peak EPSC amplitudes by mGluR agonists $(\boldsymbol{E})$ and the changes in paired-pulse ratio $(\boldsymbol{F})$ for PF-EPSCS, illustrated as in $\boldsymbol{B}$ and $\boldsymbol{C}$, respectively. $\operatorname{In} \boldsymbol{B}$ and $\boldsymbol{E}$, the numbers of tested $\mathrm{PC}$ s are indicated in parentheses. Each data point corresponds to the mean \pm SEM. 


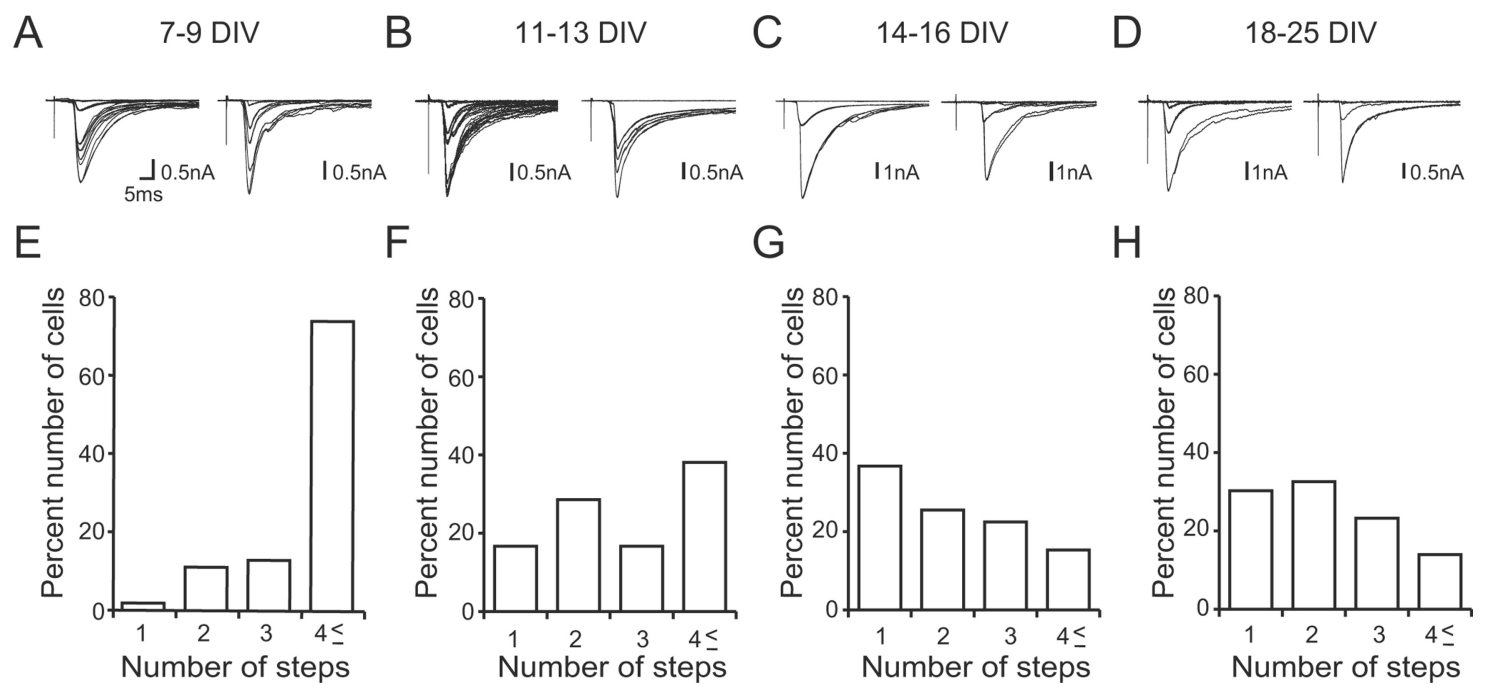

Figure 7. Developmental changes in CF-EPSCs in cocultures. $\boldsymbol{A}-\boldsymbol{D}$, Sample traces of CF-EPSCs recorded from PCs at 7-9 DIV $(\boldsymbol{A}), 11-13$ DIV (B), $14-16$ DIV (C), and $18-25$ DIV (D). Records from two representative $P(s$ are shown for each culture period. Two to three traces are superimposed at each threshold intensity. CFs were stimulated in the medullary explant. The holding potential was $-30 \mathrm{mV}$. $\boldsymbol{E}-\boldsymbol{H}$, Summary histograms showing the number of discrete steps of CF-EPSCs from cocultured PCs at 7-9 DIV (E; 54 PCs from 16 slices), 11-13 DIV (F; 42 PCs from 12 slices), $14-16$ DIV (G; $98 \mathrm{PC}$ from 26 slices), and $18-25 \mathrm{DIV}$ ( $\boldsymbol{H} ; 43 \mathrm{PC}$ from 13 slices). A clear leftward shift is noted in the distribution of the step number during coculture ( $p<0.001$ for $7-9$ DIV vs $11-13$ DIV; $p<0.01$ for 11-13 DIV vs $14-16$ DIV; $p>0.05$ for $14-16$ DIV vs $18-25$ DIV; Mann-Whitney $U$ test).
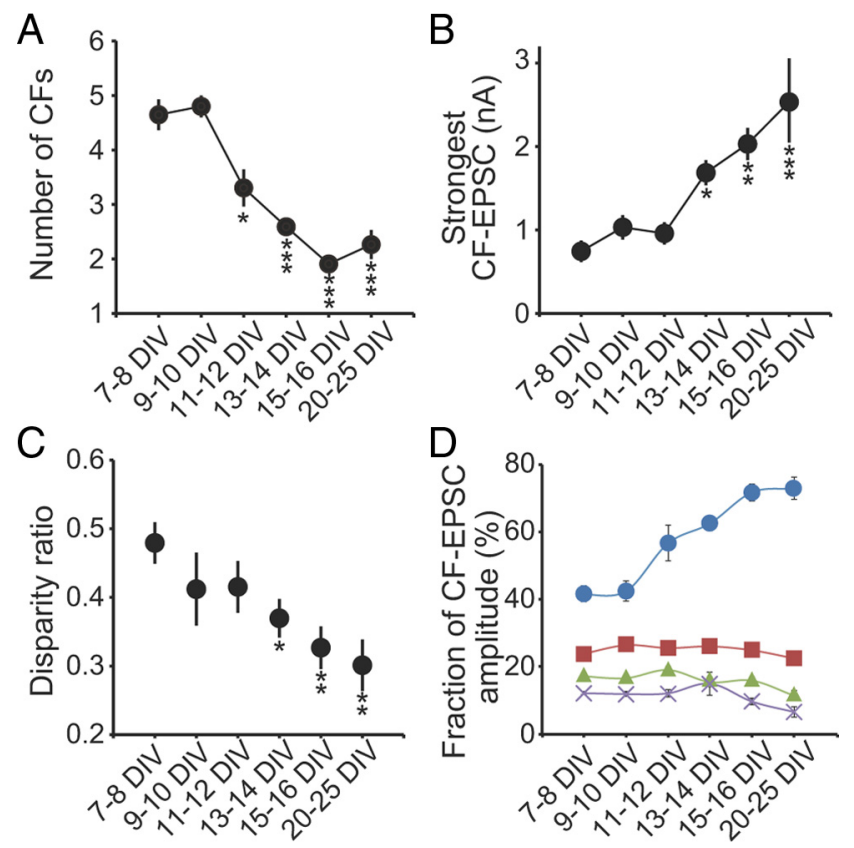

Figure 8. Changes in parameters for CF-PC synapses during coculture. $A$, Decrease in the number of $C F$ s per $P C . B$, Increase in the EPSC amplitude of the strongest $C F$ synaptic response. C, Change in the disparity among the amplitudes of multiple CF-EPSCs in individual PCs (disparity ratio). The disparity ratio was calculated as described previously (Hashimoto and Kano, 2003): disparity ratio $=\left(A_{1} / A_{N}+A_{2} / A_{N}+\cdots+A_{N-1} / A_{N}\right) /(N-1) ; N>1 . A_{i}$ is the EPSC amplitude for the $\mathrm{CF}_{i,}$ and $N$ is the number of $\mathrm{CF}$ s for a given $\mathrm{PC}$. Each data point corresponds to the mean value from $12-55$ PCs. D, Changes in the fractions of the largest (blue), second (red), third (green), and fourth (violet) EPSC amplitudes relative to the total CF-EPSC amplitude in PCS. CF-EPSCs were recorded at the same holding potential. Each data point corresponds to the mean \pm SEM. Asterisks indicate significant differences between PCs analyzed at 7-8 DIV and those at each culture period $\left({ }^{*} p<0.05 ;{ }^{* *} p<0.01 ;{ }^{* * *} p<0.001\right.$; Steel-Dwass test).

come larger relative to those of other CFs in individual PCs during postnatal development (Hashimoto and Kano, 2003; Hashimoto et al., 2011). To check whether such selective strengthening of a single $\mathrm{CF}$ among multiple CFs occurs in cocul-
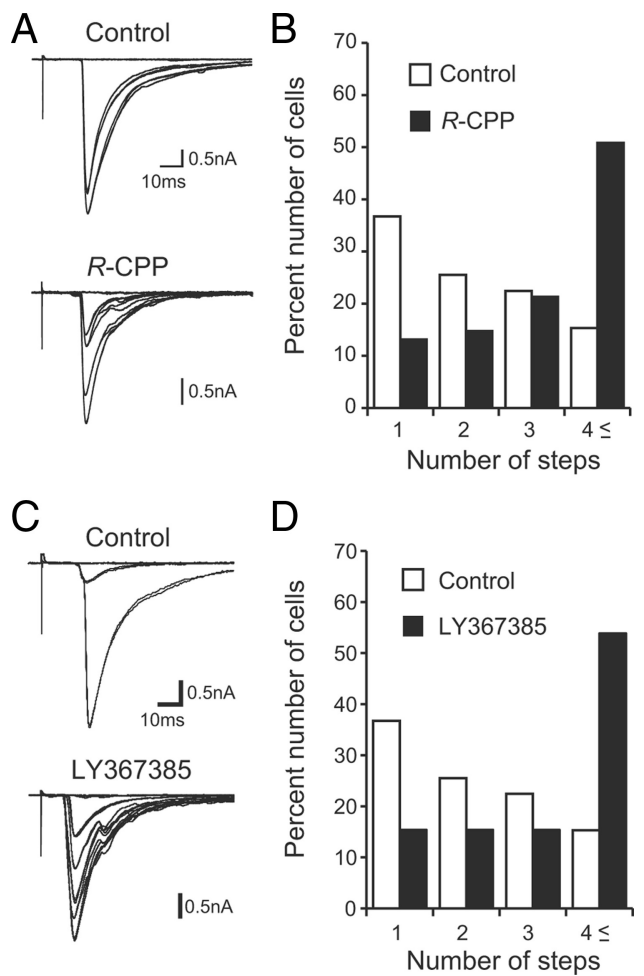

Figure 9. Effects of pharmacological manipulations on the CF synapse elimination in cocultures. $A, C$, Representative traces of CF-EPSCS recorded from PCS in control cocultures (top) or in cocultures (bottom) treated with $10 \mu \mathrm{m} R$-CPP ( $\boldsymbol{A}$ ) or $100 \mu \mathrm{m} \mathrm{L} Y 367385$ (C). Two to four traces are superimposed at each threshold stimulus intensity. The holding potential was $-30 \mathrm{mV} . \boldsymbol{B}, \mathbf{D}$, Histograms showing the number of discrete steps of CF-EPSCs for $R$-CPP ( $\boldsymbol{B} ; 98$ PCs from 26 slices for control; 71 PCs from 20 slices for $R$-CPP; $p<0.001$, Mann-Whitney $U$ test) or for LY367385 (D; 98 PCs from 26 slices for control; 33 PCs from 9 slices for LY367385; $p<0.001$, Mann-Whitney $U$ test).

tures, we calculated the disparity ratio for each multiply innervated PCs, which has been used for estimating relative strengths of CF inputs (Hashimoto and Kano, 2003; Hashimoto et al., 2011). We found that the disparity ratio decreased gradually dur- 
ing the elimination of CFs as the EPSC amplitude of the strongest CF progressively increased (Fig. $8 B, C$ ). Moreover, when the fractions of individual CF-EPSC amplitudes relative to the total CF-EPSC amplitude in each PC were calculated, the fraction of the largest CF-EPSC increased from 11 to 12 DIV, but those of the second to fourth largest CF-EPSCs remained unchanged or gradually decreased (Fig. 8D). These data indicate that multiple CFs initially have relatively similar strengths, and then single CFs become progressively stronger in individual PCs.

\section{Pharmacological manipulations of CF synapse elimination}

We addressed whether the mechanisms underlying refinement of CF-PC synaptic connections in cocultures are identical to those in the cerebellum in vivo. Previous studies have demonstrated that chronic application of NMDA receptor antagonists into developing cerebellum in vivo blocks CF synapse elimination (Rabacchi et al., 1992; Kakizawa et al., 2000). To examine whether NMDARs are required for the CF synapse elimination in cocultures, we added an NMDA receptor antagonist, $R$-CPP $(10 \mu \mathrm{M})$, in the culture medium from 7 DIV to the days of recording (after 14 to 16 DIV). We found that the NMDA antagonist induced a clear rightward shift toward higher number of CF-EPSC steps in the frequency distribution histogram of PCs (Fig. $9 A, B)(n=98$ for control; $n=$ 71 for $R$-CPP; $p<0.001$, Mann-Whitney $U$ test).

It is known that mGluR1 and the downstream signaling involving $\mathrm{G} \alpha \mathrm{q}$, phospholipase $\mathrm{C} \beta 4$, and protein kinase $\mathrm{C} \gamma$ in PCs are essential for CF synapse elimination (Kano et al., 1995, 1997, 1998; Offermanns et al., 1997; Ichise et al., 2000). We therefore tested the effect of the mGluR1-selective antagonist LY367385 $(100 \mu \mathrm{M})$ on CF synapse elimination in cocultures. As shown in Figure 9, $C$ and $D$, LY367385 applied from 7 DIV to the days of recording (after 14 to 16 DIV) caused a significant rightward shift in the frequency distribution of PCs against CFEPSC steps compared to the vehicletreated control cocultures $(n=98$ for control; $n=33$ for LY367385; $p<0.001$, Mann-Whitney $U$ test). These results indicate that mGluR1 is required for $\mathrm{CF}$ synapse elimination in cocultures. The two lines of evidence from the pharmacological blockade of NMDARs and mGluR1 indicate that CF synapse elimination in cocultures shares similar molecular mechanisms to those in vivo. In addition, the
A

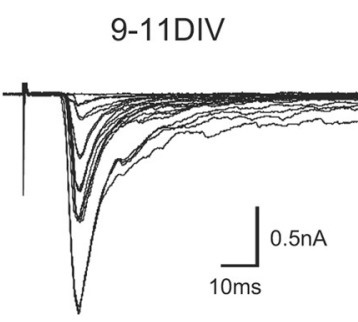

15-18DIV

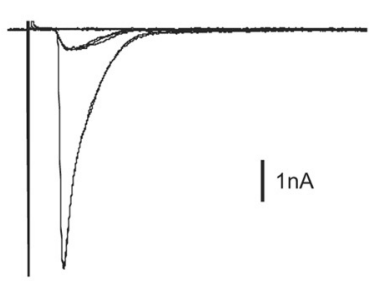

C

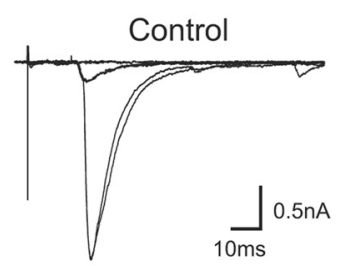

GluRס2 KO

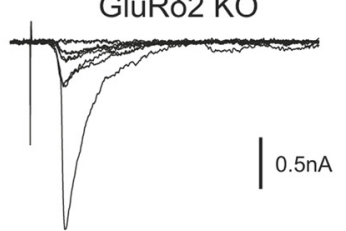

GluRס2-rescue

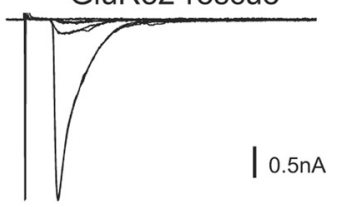

$\mathrm{B}$

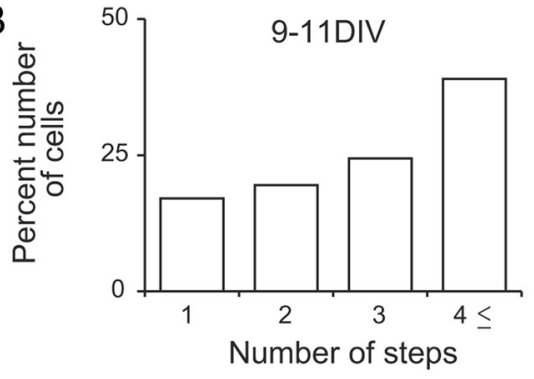

15-18DIV

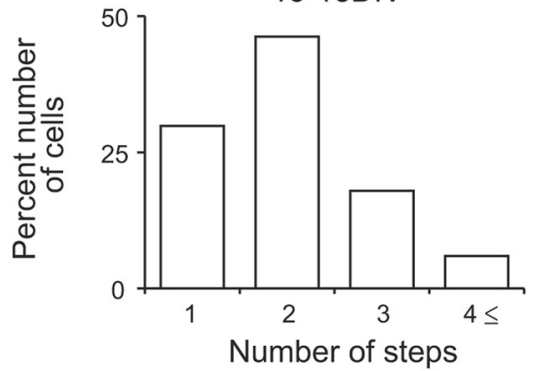

D

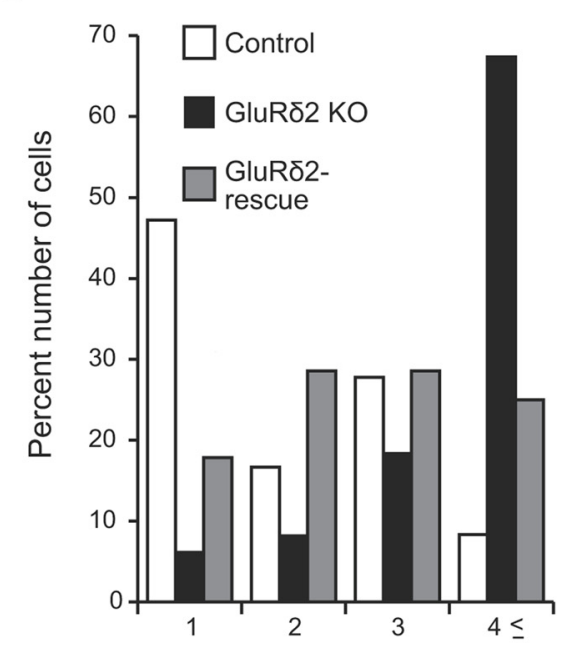

Number of steps
E

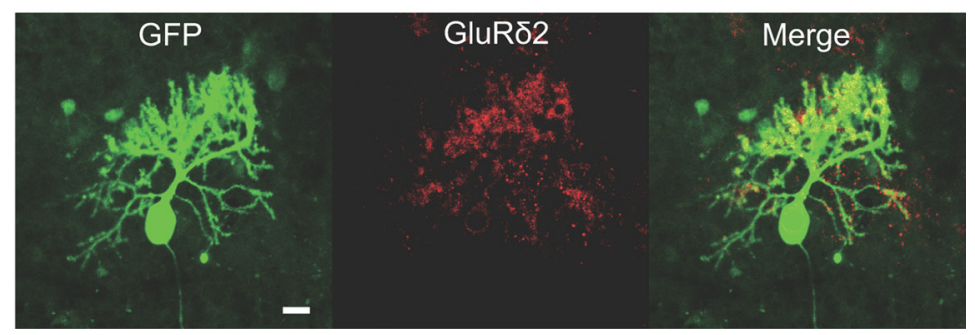

Figure 10. CF synapse elimination in chimera cocultures using GluR $\delta 2$ knock-out mice. $A, B, C F$ synapse elimination in cocultures of wild-type mouse cerebellar slices and rat medullary explants. A, CF-EPSCs recorded in PCs at 9-11 DIV and 15-18 DIV. CFs were stimulated in the medullary explant. Two to four traces are superimposed at each threshold stimulus intensity. The holding potential was $-30 \mathrm{mV}$. $\boldsymbol{B}$, Summary histograms showing the number of discrete steps of CF-EPSCs from cocultured PCsat 9-11 DIV (41 PCs from 10 slices) and 15-18 DIV (67 PCs from 18 slices). Approximately 40\% of PCs had more than four CF-EPSC steps at 9-11 DIV. At 14-16 DIV, the percentage of PCs with more than four CFs decreased, and $\sim 70 \%$ of PCs were innervated by single or two CFs ( $p<0.001$ for $9-11$ DIV vs $15-18$ DIV; Mann-Whitney $U$ test). C, Representative traces of CF-EPSCs in cocultures of cerebellar slices prepared from wild-type mice (top; control),

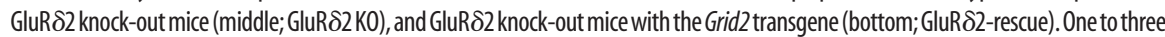
traces are superimposed at each threshold stimulus intensity. The holding potential was $-30 \mathrm{mV}$. D. Histograms showing the number of discrete steps of (F-EPSCs in control (open columns; 36 PCs from 11 slices), GluR $\delta 2$ KO (filled columns; 49 PCs from 15 slices), and the GluR $\delta 2$-rescue (hatched columns; 28 PCs from 7 slices) cerebella ( $p<0.001$ for control vs GluR $\delta 2$ K0; $p<0.001$ for GluR $\delta 2$ KO vs GluR $\delta 2$-rescue; Steel-Dwass test). E, Double immunostaining for GFP (green) and GluR $\delta 2$ (red) in a coculture of GluR $\delta 2$ KO cerebellar slices transferred with the Grid2 transgene. Scale bar: $20 \mu \mathrm{m}$. 
results clearly demonstrate that pharmacological manipulations can be reliably applied in cocultures.

\section{Genetic manipulations of CF synapse elimination}

Although pharmacological manipulations are useful, drugs added to the culture medium affect both the cerebellum and the medullary explant. To examine how the defect of a certain molecule in the cerebellum affects CF synapse elimination, we developed a chimera coculture system that was composed of the cerebellar slices from mutant mice defective in a certain molecule and the medullary explants from normal rats. First, we established a coculture system composed of a cerebellar slice from wild-type mouse and a medullary explant from rat. In this mouse-rat chimera coculture, the majority of PCs were innervated by four or more CFs at 9-11 DIV (Fig. 10A,B). The number of CFs innervating each PC decreased significantly at 15-18 DIV (Fig. $10 A, B)(n=41$ for $9-11$ DIV; $n=67$ for $15-18$ DIV; $p<$ 0.001; Mann-Whitney $U$ test). These data indicate that massive elimination of CFs occurs also in mouse-rat chimera cocultures.

We also tried to culture a cerebellar slice from mice at $\sim \mathrm{P} 9$ with a medullary explant dissected from mouse embryos at E12E15. However, it was difficult to handle small pieces of the medulla oblongata taken from mouse embryos, and the success rate of establishing CF reinnervation onto PCs in the mouse-mouse cocultures was significantly low. Therefore, in the following experiments of using cerebellar slices from mutant mice, we adopted the mouse-rat chimera coculture system.

We made cocultures using cerebellar slices from GluR $\delta 2$ knock-out mice. GluR $\delta 2$ is expressed specifically in PCs and molecular layer interneurons (Yamasaki et al., 2011), and phenotypes of GluR $\delta 2$ knockout mice have been well studied (Kashiwabuchi et al., 1995; Kurihara et al., 1997; Hashimoto et al., 2001; Lalouette et al., 2001). They exhibit severe defects in PF synapse formation and CF synapse elimination. In chimera cocultures composed of a cerebellar slice from GluR $\delta 2$ knockout mouse and a medullary explant from normal rat, the majority of PCs were innervated by four or more CFs, whereas nearly $50 \%$ of PCs were monoinnervated in cocultures of wild-type mouse cerebellum and normal rat medulla (control) (Fig. 10C,D) $(n=36$ for control; $n=49$ for GluR $\delta 2 \mathrm{KO} ; p<0.001$; Steel-Dwass test). This result clearly indicates that CF synapse elimination in chimera cocultures is impaired when GluR $\delta 2$ is absent in the cerebellum.

We further asked whether the defect in CF synapse elimination could be restored by introducing the Grid 2 transgene into PCs of the GluR $\delta 2$ knock-out cerebellum in chimera cocultures. We produced a recombinant nonreplicative lentiviral vector encoding GluR $\delta 2$ plus GFP that could be expressed under the control of a PC specific promoter, L7 (pCL20c-trL7GluR82trL7GFP). The lentiviral vector was infected into the cultured cerebellar slices from GluR $\delta 2$ knock-out mice at 1 DIV.

Notably, GluR $\delta 2$ signals were clearly observed in GFPpositive PCs transduced with pCL20c-trL7GluR $\delta 2$-trL7GFP in GluR $\delta 2$ knock-out slices (Fig. 10E). When examined at 15-18 DIV in GluR $\delta 2$ knock-out slices, the frequency distribution of PCs against the number of CF-EPSC steps showed a significant leftward shift for GluR $\delta 2$-rescued PCs compared with that for nontransduced GluR $\delta 2$ knock-out PCs (Fig. 10C,D) $(n=49$ for GluR $\delta 2 \mathrm{KO} ; n=28$ for GluR $\delta 2$ rescue; $p<0.001$; Steel-Dwass test). These results clearly indicate that GluR $\delta 2$ functions in PCs to influence CF synapse elimination, which is consistent with a previous report (Hirai et al., 2005). The results indicate that the phenotypes of GluR $\delta 2$ knock-out cerebellum that was cocultured with rat medulla are essentially the same as those found in the cerebellum of GluR $\delta 2$ knock-out mice in vivo and in acute slices.
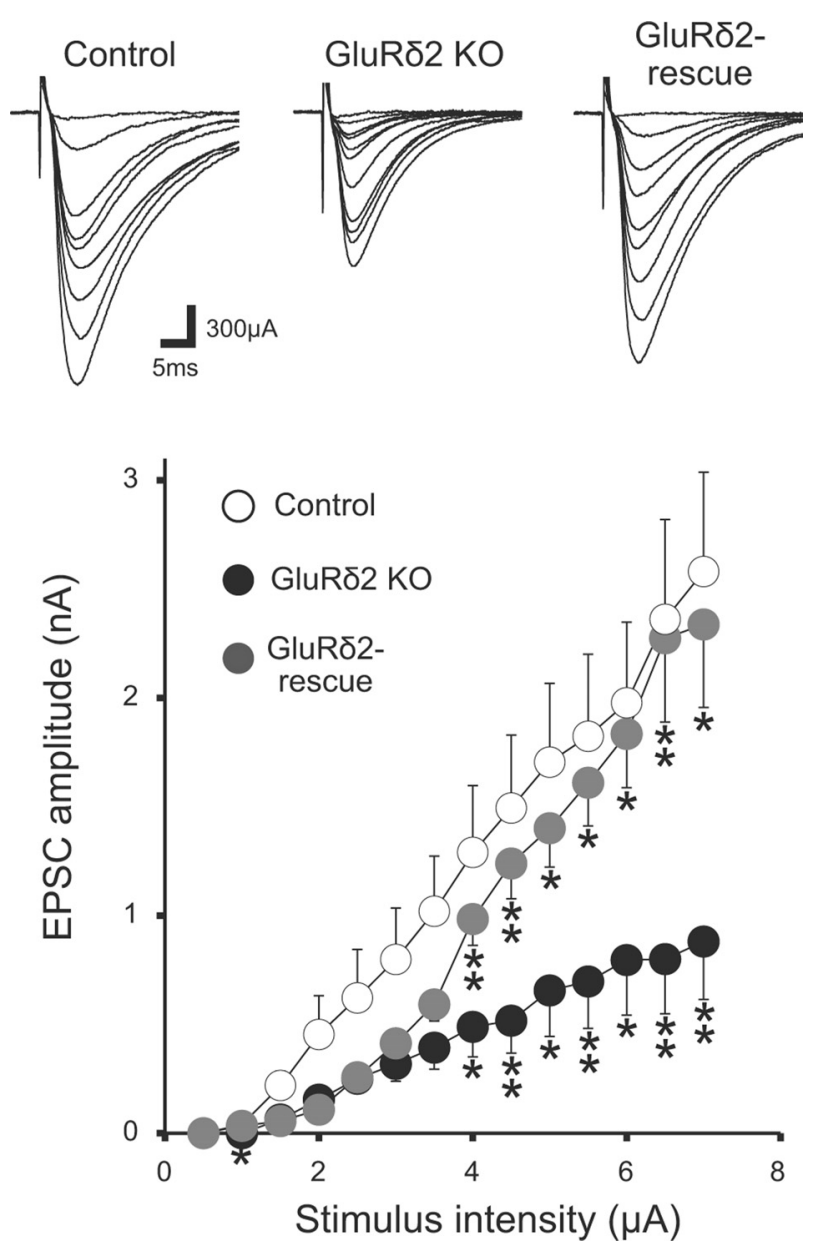

Figure 11. PF-PC synapses in chimera cocultures using GluR $\delta 2$ knock-out mice. Representative traces of PF-EPSCs with increasing stimulus intensities (top) and averaged input- output relations of PF-EPSCs (bottom) in control (13 PCs from 5 slices), GluR $\delta 2$ KO (12 PCs from 5 slices), and the GluR $\delta 2$ rescue (11 PCs from 4 slices) cocultures. The holding potential was $-80 \mathrm{mV}$. Asterisks in GluR $\delta 2 \mathrm{~K} 0$ and GluR $\delta 2$ rescue indicate significant differences when compared with control and GluR $\delta 2 \mathrm{KO}$, respectively ( ${ }^{*} p<0.05$; ${ }^{* *} p<0.01$; Steel-Dwass test).

Thus, the mouse-rat chimera coculture system developed here would be a useful preparation for studying roles of candidate molecules in the cerebellum in CF synapse elimination.

GluR $\delta 2$ is known to be crucial for the maintenance of PF-PC synapses (Kurihara et al., 1997). It was shown previously that GluR $\delta 2$ on PC spines binds to Cbln1 (Matsuda et al., 2010) that interacts with neurexin on PF terminals (Uemura et al., 2010). In mouse-rat chimera cocultures, we compared the input-output relationship of PF-EPSCs among control, GluR $\delta 2$-knock-out, and GluR $\delta 2$-rescued PCs. We found that the amplitude of PF-EPSC in GluR $\delta 2$-knock-out PCs was approximately one-third of that in control PCs, whereas the amplitude in GluR $\delta 2$-rescued PCs was nearly comparable to that in control PCs (Fig. 11). These data suggest that GluR $\delta 2$ exerts its effects on the maintenance of PF-PC synapses in chimera cocultures as well as in the cerebellum in vivo.

\section{Gain- and loss-of-function analyses of CF synapse elimination in cocultures}

We then tested whether overexpression or knockdown of certain molecules in cocultures can be applicable to the study of CF synapse elimination. We performed both gain-of-function and loss-offunction analyses of GluR $\delta 2$. We used pCL20c-trL7GluR $\delta 2-$ trL7GFP to cause overexpression of GluR 22 in wild-type PCs. To 

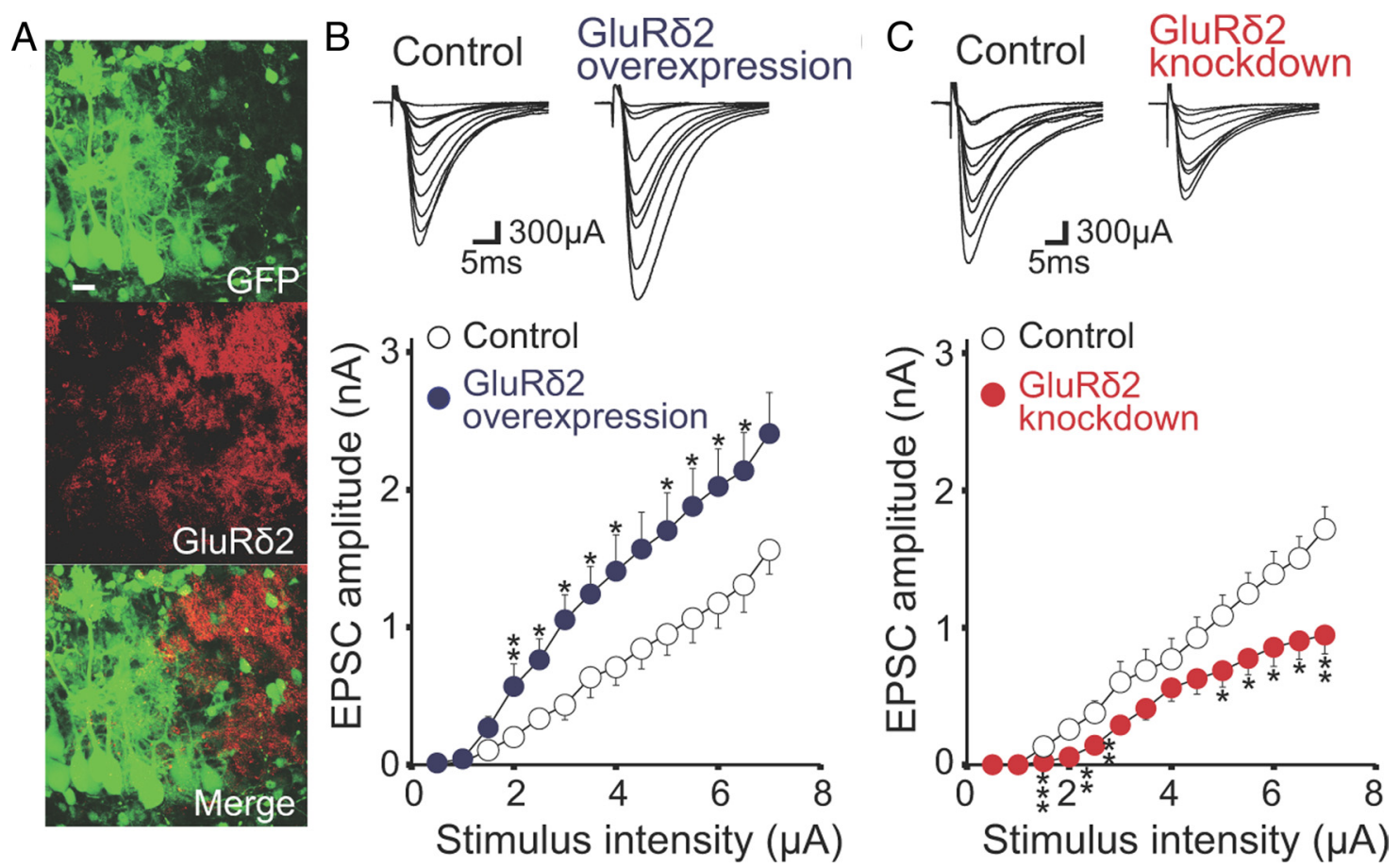

Figure 12. Gain- and loss-of-function analyses of GluR $\delta 2$ gene in cocultures. $A$, Double immunostaining for GFP (green) and GluR $\delta 2$ (red) in a coculture transferred with GFP and microRNA for GluR $\delta 2$. Scale bar: $20 \mu \mathrm{m}$. B, Representative traces (top) and averaged input- output relationship (bottom) of PF-EPSCs from control (open circles; 4 slices, $12 \mathrm{PCs}$ ) and GluR $\delta 2$-overexpressing (blue circles; 4 slices, $14 \mathrm{PCs}$ ) cocultures. C, Representative traces (top) and averaged input- output relationship (bottom) of PF-EPSCs from control (open circles; 4 slices, $16 \mathrm{PCs}$ ) and GluR $22 \mathrm{knockdown}$ (red circles; 4 slices, $16 \mathrm{PC}$ ) cocultures. The holding potential was $-80 \mathrm{mV}$. Asterisks indicate significant differences when compared with control $\left({ }^{*} p<0.05 ;{ }^{* *} p<0.01\right.$; ${ }^{* * *} p<0.005$; Mann-Whitney $U$ test).

knock down GluR $\delta 2$, we produced a lentiviral vector encoding miR designed against GluR $\delta 2$ and GFP under the control of trL7 promoter (pCL20c-trL7-GluR $\delta 2$ miR-GFP). Both lentiviral vectors were infected to mouse cerebellar slices cocultured with rat medullary explants at $1 \mathrm{DIV}$, and GluR $\delta 2$ expression was examined after $8 \mathrm{~d}$ in cocultures by immunohistochemistry. GluR $\delta 2$ labeling was dramatically reduced in PCs transduced with pCL20c-trL7GluR $\delta 2 \mathrm{miR}-\mathrm{GFP}$, but not in untransduced PCs (Fig. 12A).

We tested whether the GluR $\delta 2$ overexpression and knockdown affected maintenance of PF-PC synapses by measuring the inputoutput relationship of PF-EPSC amplitudes. We found that PFEPSCs of PCs with GluR $\delta 2$ overexpression (GFP-positive PCs) were larger than those of control untransduced PCs (GFP-negative PCs) taken in the same cocultures (Fig. 12B). In contrast, PF-EPSCs of PCs with GluR $\delta 2$ knockdown (GFP-positive PCs) were significantly smaller than those of control untransduced PCs (GFP-negative PCs) taken in the same cocultures (Fig. 12C).

We then examined whether overexpression and knockdown of GluR $\delta 2$ affect CF synapse elimination in cocultures. At 15-18 DIV, frequency distribution of PCs against the number of CF-EPSC steps showed a significant leftward shift for PCs with GluR $\delta 2$ overexpression compared with control nontransduced PCs taken from the same cocultures (Fig. $13 A, B)(n=57$ for control; $n=43$ for GluR $\delta 2$ overexpression; $p<0.05$; Mann-Whitney $U$ test). Conversely, the distribution exhibited a significant rightward shift for PCs with GluR $\delta 2$ knockdown compared with control nontransduced PCs from the same cocultures (Fig. 13C,D) $(n=52$ for control; $n=30$ for GluR $\delta 2$ knockdown; $p<0.05$; Mann-Whitney $U$ test). These results indicate that overexpression and knockdown of GluR $\delta 2$ promoted and suppressed, respectively, the CF synapse elimination in cocultures. Together, the present results suggest that the functions of GluR $\delta 2$ in cocultures are similar to those found in vivo. Thus, the coculture is useful for both gain- and loss-of-function analyses for CF synapse elimination.

\section{Identification of a new molecule involved in CF synapse elimination by using the coculture preparation}

Finally, we performed functional assay of new candidate molecules required for $\mathrm{CF}$ synapse elimination by using the coculture preparation. Neuroligins are expressed on the surface of postsynaptic membranes at both excitatory and inhibitory synapses and play key roles in the formation, maturation and functions of synapses (Scheiffele et al., 2000; Varoqueaux et al., 2006; Chubykin et al., 2007). Importantly, the expression level of neuroligins increases with a twofold to threefold enhancement during the period when CF-PC synapse elimination occurs (Varoqueaux et al., 2006). However, the role of neuroligins in synapse elimination has not yet been reported. We focused on neuroligin-2 that are highly expressed in PCs (Allen Brain Atlas; http://mouse.brain-map.org) and generated PCspecific lentiviral knockdown vectors of neuroligin-2. We first tested the effectiveness of our neuroligin-2 knockdown vector by assessing its potency to suppress the heterologous expression of neuroligin-2 in HEK293T cells. When compared to HEK293T cells cotransduced with a GFP expression control vector and a neuroligin-2 expression vector, the expression level of neuroligin-2 was markedly reduced in HEK293T cells cotransduced with the neuroligin-2 knockdown vector and the neuroligin- 2 expression vector (Fig. $14 A, B)(n=8$ for GFP control; $n=9$ for neuroligin-2 knockdown; $p<0.001$; SteelDwass test). Then, the knockdown vector was transduced into PCs of the olivocerebellar coculture, the target molecule was knocked down in a PC-specific manner, and CF synapse elimination was examined. We found that knockdown of neuroligin-2 in PCs significantly impaired CF synapse elimination (Fig. 14C,D) $(n=76$ for control; $n=45$ for neuroligin-2 knockdown; $p<0.05$; Steel-Dwass 

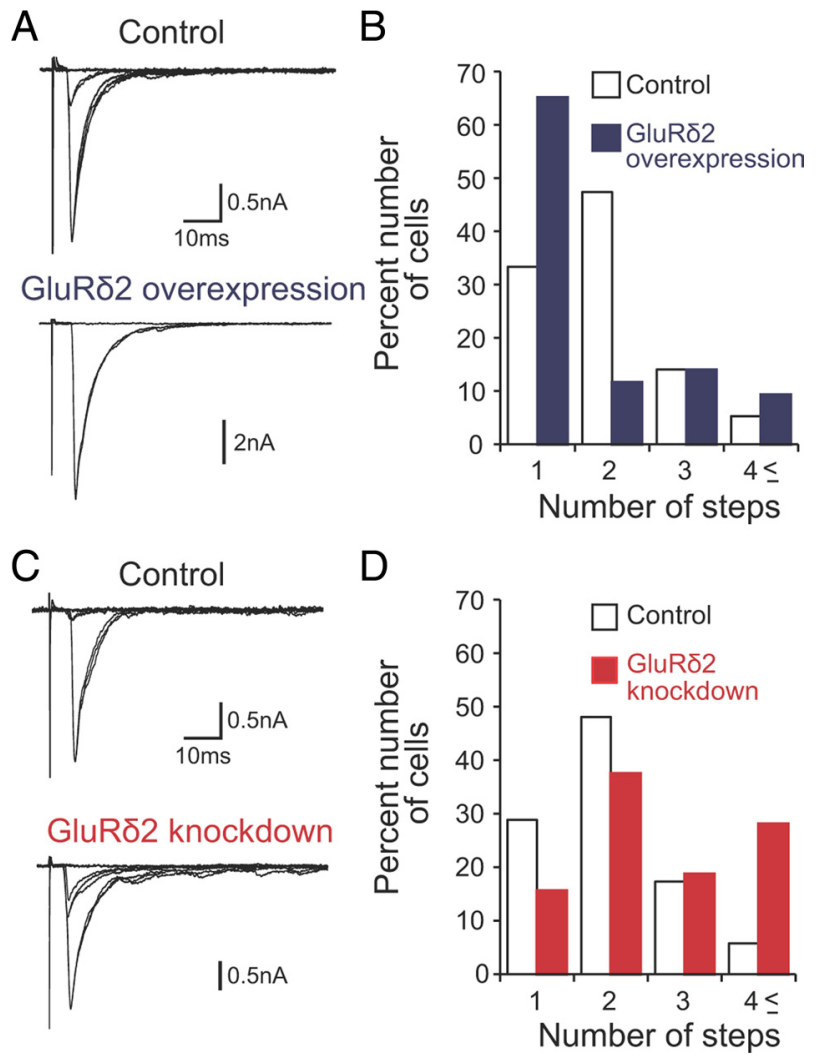

Figure 13. CF synapse elimination in gain- and loss-of-function analyses of GluR $\delta 2$ gene in cocultures. $A, B$, Representative traces of CF-EPSCS $(\boldsymbol{A})$ and frequency distribution histograms showing the number of discrete steps of CF-EPSCS ( $\boldsymbol{B}$ ) for control (open columns; 57 PCs from 18 slices) and GluR $\delta 2$-overexpressing (blue columns; 43 PCs from the same slices in which control PCs were sampled) cocultures ( $p<0.05$; Mann-Whitney $U$ test). Two to four traces are superimposed at each threshold stimulus intensity. The holding potential was $-30 \mathrm{mV}$. C, D, Representative traces of (FEPSCS ( $C$ and frequency distribution histograms ( $D$ ) for control (open columns; $52 \mathrm{PCs}$ from 10 slices) and GluR $\delta 2$ knockdown (red columns; 30 PCsfrom the same slices in which control PCs were sampled) cocultures ( $p<0.05$; Mann-Whitney $U$ test). Two to four traces are superimposed at each threshold stimulus intensity. The holding potential was $-30 \mathrm{mV}$.

test). In contrast, the neuroligin-2 knockdown did not change the total amplitude and disparity of CF-EPSCs in individual PCs (Table 1). The effect of neuroligin-2 knockdown was largely rescued by an RNAi-resistant neuroligin-2 cDNA (neuroligin-2 RES), in which seven nucleotides were mutated without changing the amino acid sequence in the RNAi targeted site. When neuroligin-2 RES was cotransfected with the neuroligin-2 knockdown vector in HEK293T cells, the expression of neuroligin-2 was restored to the control level (Fig. $14 A, B)(n=8$ for GFP control; $n=9$ for neuroligin-2 knockdown; $n=9$ for neuroligin-2 RES; $p<0.001$; Steel-Dwass test). The transduction of the neuroligin-2 RES with neuroligin-2 knockdown in cocultures caused a significant leftward shift in the frequency distribution of PCs against CF-EPSC steps compared with neuroligin-2 knockdown (Fig. 14C,D) $(n=45$ for neuroligin-2 knockdown; $n=$ 39 for neuroligin-2 RES; $p<0.005$; Steel-Dwass test). These results strongly suggest that neuroligin- 2 in postsynaptic $P C$ is required for CF synapse elimination. Thus, the olivocerebellar coculture preparation is useful for screening new candidate molecules that are involved in CF synapse elimination.

\section{Discussion}

Cultures have several advantages over in vivo preparations, such as the accessibility and flexibility of various experimental manipulations, which enable us to perform pharmacological and genetic manipulations in temporally and spatially controlled manners. Cultures of dissociated neurons have been used for an unbiased screening of molecules such as those involved in synapse formation (Umemori et al., 2004; Paradis et al., 2007). However, it is difficult to examine the development of specific circuits because neurons in dissociated cultures do not retain cytoarchitecture and neural circuits of the brain tissues in vivo. Organotypic culture preparations have also been used for the study of synapse development and plasticity (Yamamoto et al., 1989; Stoppini et al., 1991; Gähwiler et al., 1997). The preparations preserve the well-defined cellular architecture of the in vivo neural circuit, which allows the identification and manipulation of defined neurons and synapses. Since the organotypic cultures have essentially the same advantages over in vivo preparations as dissociated cultures, the preparations should be suitable for a large-scale screening of molecules involved in synapse formation, maturation, and elimination.

The organotypic olivocerebellar coculture preparation described here provides a fast, efficient, and flexible experimental system to study the mechanisms of synapse elimination in the mammalian CNS. The coculture has clear advantages over experimental systems in vivo. First, the viral vectors with relatively low biological titers (lower than $10^{8} \mathrm{TU} / \mathrm{ml}$ ) are sufficient for the analyses in cocultures. Second, pharmacological and genetic manipulations including overexpression and knockdown could be easily and efficiently applied in a temporally and spatially controlled manner. An experienced investigator can prepare $>10$ lentiviral vectors for overexpression and knockdown in a week and can examine the functions of genes of interest in a few weeks. Such a fast and efficient analysis is impossible by using transgenic and knock-out animals in vivo. Third, manipulation of multiple molecules in the same cells can be applicable in cocultures. The genetic manipulation combined with application of bioactive proteins will be useful for evaluating the functional coupling and concerted actions of different proteins.

Previous studies of olivocerebellar slice cultures have shown that CF-PC synapses can be formed in vitro (Knöpfel et al., 1990; Mariani et a., 1991). In addition to synapse formation, we demonstrate that CF synapse elimination occurs in our olivocerebellar cocultures, which involves the same molecules that are known to be required for CF synapse elimination in vivo. Although the developmental changes in CF-PC synapses in cocultures are largely similar to those found in vivo, there exist some clear differences. While only $\sim 20 \%$ of PCs are innervated by multiple CFs in the cerebellum of mice older than P21 (Kano et al., 1997, 1998; Offermanns et al., 1997), 60\% of PCs are innervated by multiple CFs in cocultures (Fig. 7C,D). In addition, while selective strengthening of single CFs in individual PCs precede elimination of redundant CFs in vivo (Hashimoto and Kano, 2003; Hashimoto et al., 2009b; Kano and Hashimoto, 2009; Watanabe and Kano, 2011), these two events proceed in parallel in cocultures (Fig. $8 A, C$ ). These differences may be attributable to the technical limitations related to the preparation of cocultures. We found that CFs could hardly grow from the medulla into the cerebellum when the medullary explants taken from rat embryos older than E15-E16 were used. In the previous study of culturing medullary explants with cerebellar cells, Hirano (1990) also used medullary explants taken from E16 rats and demonstrated that stimulation of the medullary explants elicited all-or-none responses in PCs that resembled climbing fiber responses. On the other hand, the layer structure of the cerebellum and morphology of PCs were disturbed significantly when we used cerebellar slices from rats or mice at ages other than around P9. Therefore, there is a mismatch between the developmental stage of the inferior 


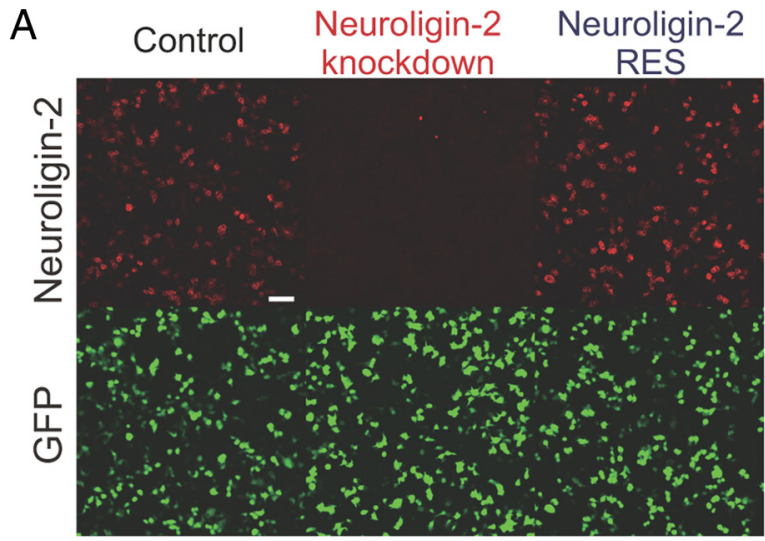

B
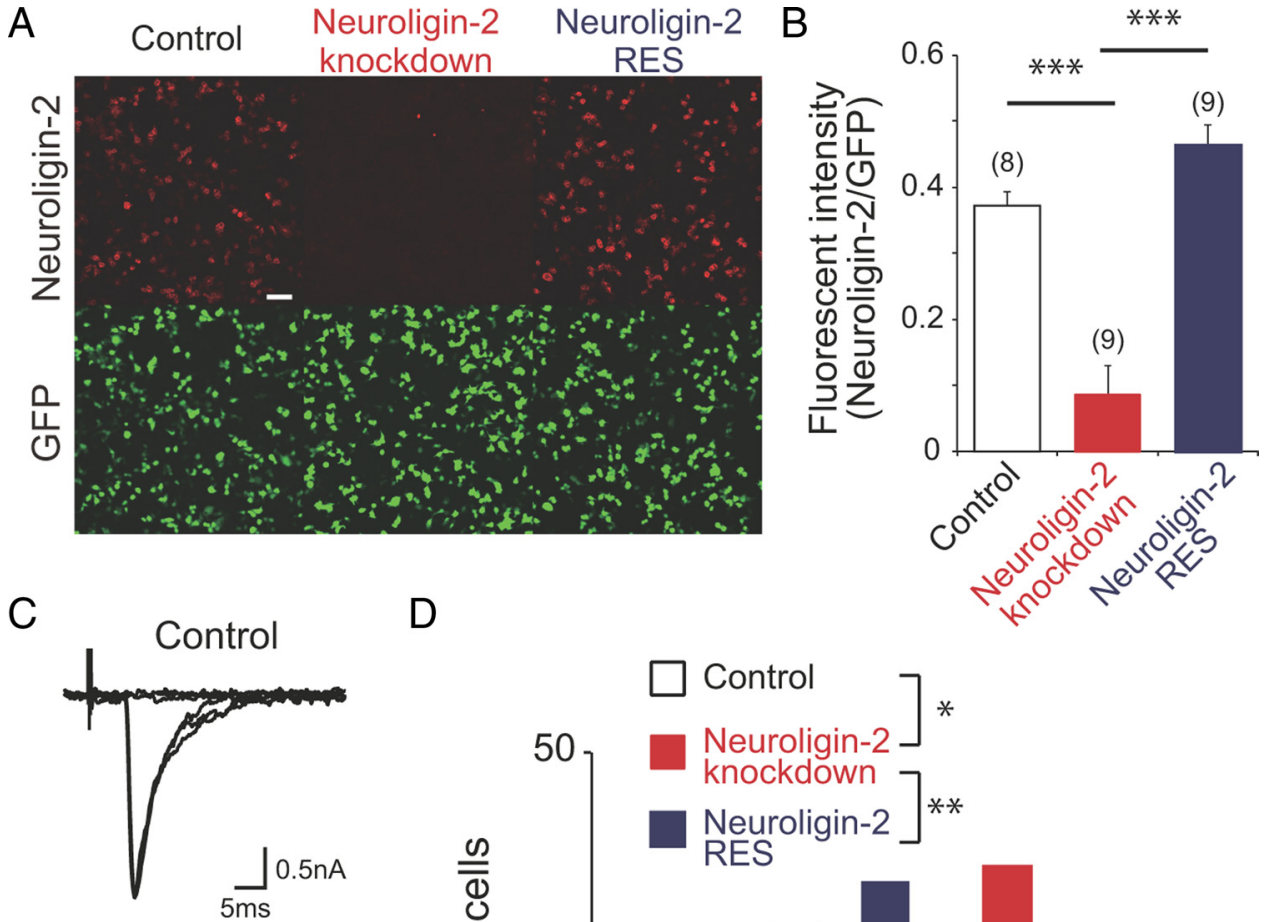

D

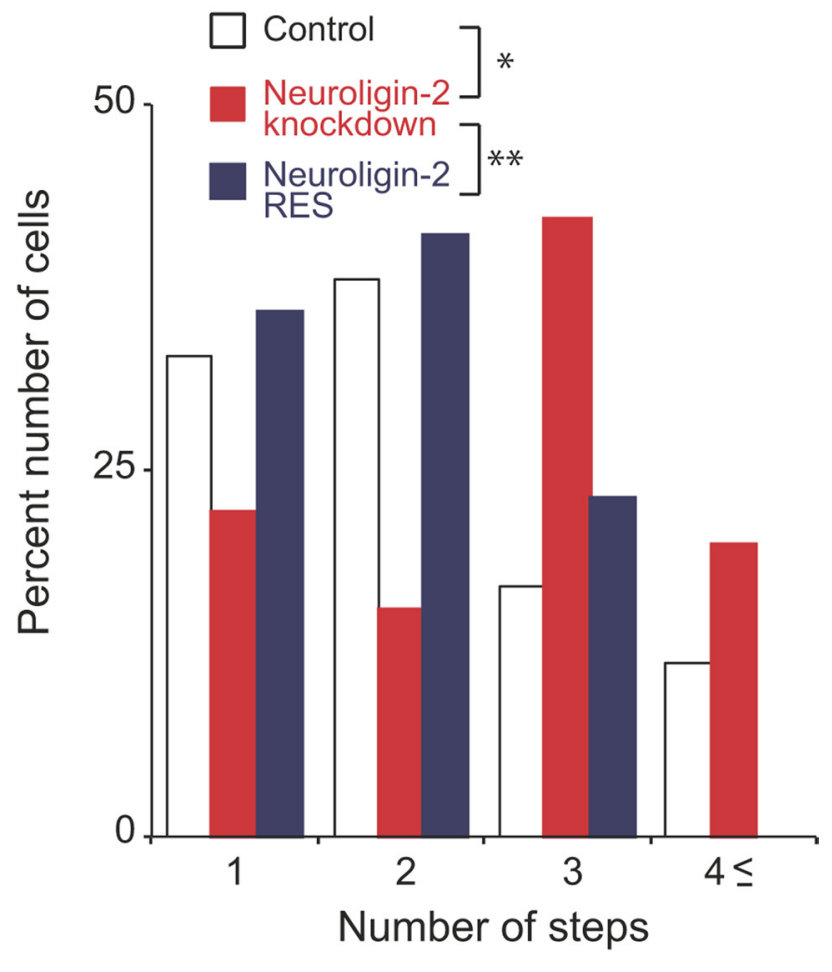

Figure 14. Impaired CF synapse elimination in cocultures with neuroligin-2 knockdown. $A$, Efficacy of neuroligin-2 knockdown by a microRNA vector. Double immunostaining for neuroligin-2 (red) and GFP (green) in HEK293T cells transfected simultaneously with a neuroligin-2 expression vector and a GFP expression vector (left; control), with the neuroligin-2 expression vector and the microRNA vector for neuroligin-2 (middle; neuroligin-2 knockdown) or with an RNAi-resistant neuroligin-2 expression vector and the microRNA vector for neuroligin-2 (right; neuroligin-2 RES). Scale bar: $30 \mu \mathrm{m}$. B, Fluorescent intensity of neuroligin-2 relative to that of GFP for control (open column), neuroligin-2 knockdown (red column), and neuroligin-2 RES (blue column; ${ }^{* * *} p<0.001$, Steel-Dwass test). Numbers of tested PCs are indicated in parentheses. C, $\boldsymbol{D}$, Representative traces of (F-EPSCS $(\boldsymbol{C})$ and frequency distribution histogram showing the number of discrete CF-EPSC steps (D) for control PCs (open columns; 37 PCs from 10 slices), neuroligin-2 knockdown PCs (red columns; 37 PCs from 10 slices), and PCs with neuroligin-2 knockdown and the RNAi-resistant neuroligin-2 2 DNA (blue columns; 39 PCs from 8 slices; ${ }^{*} p<0.05,{ }^{* *} p<0.005$, Steel-Dwass test). Two to four traces are superimposed at each threshold stimulus intensity. The holding potential was $-20 \mathrm{mV}$.

Table 1. Total amplitudes and disparity ratio of CF-EPSCs in individual PCs (holding potential of $-20 \mathrm{mV}$ ) were not different in PCs with neuroligin-2 knockdown (NL2 KD) and PCs with neuroligin-2 knockdown and neuroligin-2 RES (NL2 RES)

\begin{tabular}{lll}
\hline Type & Total amplitude $(\mathrm{nA})$ & Disparity ratio \\
\hline Control & $1.59 \pm 0.16(n=55)$ & $0.41 \pm 0.03(n=47)$ \\
NL2 KD & $1.63 \pm 0.22(n=42)$ & $0.51 \pm 0.04(n=32)$ \\
NL2 RES & $1.53 \pm 0.28(n=32)$ & $0.35 \pm 0.03(n=23)$
\end{tabular}

Data are presented as mean \pm SEM. olive and that of the cerebellum in our coculture preparation. This mismatch appears to be a major reason for the differences in the developmental changes in CF-PC synapses between the cocultures and the cerebellum in vivo. However, it should be noted that the development of CF-PC synapses in cocultures involved the same molecules that are known to be required for CF synapse elimination in vivo. We therefore conclude that the olivocerebellar coculture is useful for a high-throughput screening of candidate molecules that are involved in CF synapse elimination.

In the present study, by using the olivocerebellar coculture preparation, we could identify neuroligin-2 as a novel molecule 
required for CF synapse elimination. How neuroligin-2 influences CF synapse elimination remains unknown. However, since neuroigin-2 has been shown to be involved in formation of inhibitory synapses (Prange et al., 2004; Chih et al., 2005; Chubykin et al., 2007), a likely possibility would be that neuroligin-2 contributes to the maturation of GABAergic inhibitory synapses onto PCs, and thereby influences the elimination of redundant $\mathrm{CF}$ synapses. This scenario is consistent with our recent report that GABAergic inhibition onto PC somata facilitates the elimination of weak CF synapses (Nakayama et al., 2012).

So far, only a few culture preparations for synapse refinement have been reported (Ohno and Sakurai, 2005; Letellier et al., 2009). A culture preparation of hindbrain explants containing the cerebellar plate and brainstem taken from E14 mouse has been reported for the study of CF synapse elimination (Letellier et al., 2009). In this hindbrain explant culture, PCs were initially innervated by multiple CFs, and then supernumerary CFs were eliminated as in vivo. Using a coculture experimental paradigm in which the cerebellum and brainstem were in different maturation stages, Letellier et al. (2009) showed that CF synapse elimination occurred only once during developmental maturation of PCs. Although the hindbrain explant culture is certainly useful, there are some disadvantages compared with the coculture preparation developed in the present study. For example, layer structure of the cerebellum is rather disturbed, and the development of the $\mathrm{PC}$ dendritic tree is far less extensive than in the cocultures even at 22 DIV (which corresponds to P16 in vivo). It is unknown whether the molecular mechanisms for CF synapse elimination in the hindbrain explant culture are similar to those in vivo. It is also unclear whether pharmacological and genetic manipulations can be easily applied to the hindbrain explants. Moreover, preparing chimeric olivocerebellar cocultures with cerebellar slices from various transgenic and knock-out mice would be much easier in our system than in the hindbrain explant culture system.

Lentiviral vectors used here for overexpression and knockdown in PCs are also available for various cell types because lentiviruses have a wide cellular tropism, and the RNA polymerase II-based systems allow the use of various tissue- and cell-specific promoters. Moreover, this approach might be applied to the elucidation of the mechanisms of climbing fiber synapse formation by overexpression of genes of interest and by treatment of proteins and drugs during the first week in culture, when climbing fiber synapses are actively formed. These findings imply that roles of certain molecules in synapse formation and elimination can be investigated for various neural circuits in the CNS by using this approach. In addition, since our vectors are also applicable to the brain in vivo (Torashima et al., 2006), the functions of candidate molecules in intact brain can be investigated efficiently.

In our coculture preparation, olivary neurons were spontaneously active. The result that pharmacological blockade of NMDA receptors impaired CF synapse elimination in cocultures implies that spontaneous activity at mossy fiber to granule cell synapse might be important as expected from our previous study (Kakizawa et al., 2000). Since these are no sensory or motor inputs from brain regions other than the medulla and the cerebellum in cocultures, spontaneous neural activity should play a crucial role in CF synapse elimination. Previous studies have demonstrated that spontaneous neural activity is crucial for the developmental refinement of neural circuits including the retinogeniculate and thalamocortical projection systems (Zhang and Poo, 2001; Huberman, 2007). However, the mechanisms by which spontaneous neural activity influences the refinement of neural circuit remain largely unknown.
The organotypic coculture preparations are useful tools for studying the mechanisms of activity-dependent neural circuit refinement (Ohno and Sakurai, 2005; Uesaka et al., 2007; Yamada et al., 2010). Applications of Brainbow transgenes (Livet et al., 2007), genetically encoded calcium reporter (Miyawaki et al., 1997; Mank et al., 2008), and optogenetic technologies such as channelrhodopsin-2 (Boyden et al., 2005) into specific cell types enable us to observe the development of neural circuits by time-lapse imaging and to control neural activity in a temporally and spatially controlled manner.

The accessibility and flexibility of various experimental manipulations in cocultures provide opportunities to overcome several major limitations in the study of neural circuit development. One of the important issues is how to narrow down key molecules out of thousands of candidates in fast and reliable ways. The olivocerebellar organotypic coculture preparation enables high throughput screening of candidate molecules that may be involved in CF synapse elimination and will greatly facilitate the research on neural circuit formation during postnatal development.

\section{References}

Altman J, Bayer SA (1997) Development of the cerebellar system: in relation to its evolution, structure, and functions. Boca Raton, FL: CRC.

Bosman LW, Takechi H, Hartmann J, Eilers J, Konnerth A (2008) Homosynaptic long-term synaptic potentiation of the "winner" climbing fiber synapse in developing Purkinje cells. J Neurosci 28:798-807.

Boyden ES, Zhang F, Bamberg E, Nagel G, Deisseroth K (2005) Millisecondtimescale, genetically targeted optical control of neural activity. Nat Neurosci 8:1263-1268.

Chih B, Engelman H, Scheiffele P (2005) Control of excitatory and inhibitory synapse formation by neuroligins. Science 307:1324-1328.

Chih B, Gollan L, Scheiffele P (2006) Alternative splicing controls selective trans-synaptic interactions of the neuroligin-neurexin complex. Neuron 51:171-178

Chubykin AA, Atasoy D, Etherton MR, Brose N, Kavalali ET, Gibson JR, Südhof TC (2007) Activity-dependent validation of excitatory versus inhibitory synapses by neuroligin-1 versus neuroligin-2. Neuron 54:919-931.

Crepel F (1982) Regression of functional synapses in the immature mammalian cerebellum. Trends Neurosci 5:266-269.

Gähwiler BH, Capogna M, Debanne D, McKinney RA, Thompson SM (1997) Organotypic slice cultures: a technique has come of age. Trends Neurosci 20:471-477.

Hanawa H, Kelly PF, Nathwani AC, Persons DA, Vandergriff JA, Hargrove P, Vanin EF, Nienhuis AW (2002) Comparison of various envelope proteins for their ability to pseudotype lentiviral vectors and transduce primitive hematopoietic cells from human blood. Mol Ther 5:242-251.

Hashimoto K, Kano M (2003) Functional differentiation of multiple climbing fiber inputs during synapse elimination in the developing cerebellum. Neuron 38:785-796.

Hashimoto K, Kano M (2005) Postnatal development and synapse elimination of climbing fiber to Purkinje cell projection in the cerebellum. Neurosci Res 53:221-228.

Hashimoto K, Miyata M, Watanabe M, Kano M (2001) Roles of phospholipase $\mathrm{C} \beta 4$ in synapse elimination and plasticity in developing and mature cerebellum. Mol Neurobiol 23:69-82.

Hashimoto K, Ichikawa R, Kitamura K, Watanabe M, Kano M (2009a) Translocation of a "winner" climbing fiber to the Purkinje cell dendrite and subsequent elimination of "losers" from the soma in developing cerebellum. Neuron 63:106-118.

Hashimoto K, Yoshida T, Sakimura K, Mishina M, Watanabe M, Kano M (2009b) Influence of parallel fiber-Purkinje cell synapse formation on postnatal development of climbing fiber-Purkinje cell synapses in the cerebellum. Neuroscience 162:601-611.

Hashimoto K, Tsujita M, Miyazaki T, Kitamura K, Yamazaki M, Shin HS, Watanabe M, Sakimura K, Kano M (2011) Postsynaptic P/Q-type Ca ${ }^{2+}$ channel in Purkinje cell mediates synaptic competition and elimination in developing cerebellum. Proc Natl Acad Sci U S A 108:9987-9992.

Hirai H, Miyazaki T, Kakegawa W, Matsuda S, Mishina M, Watanabe M, Yuzaki M (2005) Rescue of abnormal phenotypes of the $\delta 2$ glutamate receptor-null mice by mutant $\delta 2$ transgenes. EMBO Rep 6:90-95. 
Hirano T (1990) Synaptic transmission between rat inferior olivary neurons and cerebellar Purkinje cells in culture. J Neurophysiol 63:181-189.

Huberman AD (2007) Mechanisms of eye-specific visual circuit development. Curr Opin Neurobiol 17:73-80.

Ichise T, Kano M, Hashimoto K, Yanagihara D, Nakao K, Shigemoto R, Katsuki M, Aiba A (2000) mGluR1 in cerebellar Purkinje cells essential for long-term depression, synapse elimination, and motor coordination. Science 288:1832-1835.

Kakizawa S, Yamasaki M, Watanabe M, Kano M (2000) Critical period for activity-dependent synapse elimination in developing cerebellum. J Neurosci 20:4954-4961.

Kano M, Hashimoto K (2009) Synapse elimination in the central nervous system. Curr Opin Neurobiol 19:154-161.

Kano M, Hashimoto K, Chen C, Abeliovich A, Aiba A, Kurihara H, Watanabe M, Inoue Y, Tonegawa S (1995) Impaired synapse elimination during cerebellar development in PKC $\gamma$ mutant mice. Cell 83:1223-1231.

Kano M, Hashimoto K, Kurihara H, Watanabe M, Inoue Y, Aiba A, Tonegawa S (1997) Persistent multiple climbing fiber innervation of cerebellar Purkinje cells in mice lacking mGluR1. Neuron 18:71-79.

Kano M, Hashimoto K, Watanabe M, Kurihara H, Offermanns S, Jiang H, Wu Y, Jun K, Shin HS, Inoue Y, Simon MI, Wu D (1998) Phospholipase C $\beta 4$ is specifically involved in climbing fiber synapse elimination in the developing cerebellum. Proc Natl Acad Sci U S A 95:15724-15729.

Kashiwabuchi N, Ikeda K, Araki K, Hirano T, Shibuki K, Takayama C, Inoue Y, Kutsuwada T, Yagi T, Kang Y, Aizawa S, Mishina M (1995) Impairment of motor coordination, Purkinje cell synapse formation, and cerebellar long-term depression in GluR $\delta 2$ mutant mice. Cell 81:245-252.

Knöpfel T, Audinat E, Gähwiler BH (1990) Climbing fibre responses in olivo-cerebellar slice cultures. I. Microelectrode recordings from Purkinje cells. Eur J Neurosci 2:726-732.

Konnerth A, Llano I, Armstrong CM (1990) Synaptic currents in cerebellar Purkinje cells. Proc Natl Acad Sci U S A 87:2662-2665.

Kurihara H, Hashimoto K, Kano M, Takayama C, Sakimura K, Mishina M, Inoue Y, Watanabe M (1997) Impaired parallel fiber $\rightarrow$ Purkinje cell synapse stabilization during cerebellar development of mutant mice lacking the glutamate receptor $\delta 2$ subunit. J Neurosci 17:9613-9623.

Lalouette A, Lohof A, Sotelo C, Guénet J, Mariani J (2001) Neurobiological effects of a null mutation depend on genetic context: comparison between two hotfoot alleles of the $\delta 2$ ionotropic glutamate receptor. Neuroscience 105:443-455.

Letellier M, Wehrlé R, Mariani J, Lohof AM (2009) Synapse elimination in olivo-cerebellar explants occurs during a critical period and leaves an indelible trace in Purkinje cells. Proc Natl Acad Sci U S A 106:14102-14107.

Livet J, Weissman TA, Kang H, Draft RW, Lu J, Bennis RA, Sanes JR, Lichtman JW (2007) Transgenic strategies for combinatorial expression of fluorescent proteins in the nervous system. Nature 450:56-62.

Lohof AM, Delhaye-Bouchaud N, Mariani J (1996) Synapse elimination in the central nervous system: functional significance and cellular mechanisms. Rev Neurosci 7:85-101.

Maejima T, Hashimoto K, Yoshida T, Aiba A, Kano M (2001) Presynaptic inhibition caused by retrograde signal from metabotropic glutamate to cannabinoid receptors. Neuron 31:463-475.

Maejima T, Oka S, Hashimotodani Y, Ohno-Shosaku T, Aiba A, Wu D, Waku K, Sugiura T, Kano M (2005) Synaptically driven endocannabinoid release requires $\mathrm{Ca}^{2+}$-assisted metabotropic glutamate receptor subtype 1 to phospholipase $\mathrm{C} \beta 4$ signaling cascade in the cerebellum. J Neurosci 25:6826-6835.

Mank M, Santos AF, Direnberger S, Mrsic-Flogel TD, Hofer SB, Stein V, Hendel T, Reiff DF, Levelt C, Borst A, Bonhoeffer T, Hübener M, Griesbeck $\mathrm{O}$ (2008) A genetically encoded calcium indicator for chronic in vivo two-photon imaging. Nat Methods 5:805-811.

Mariani J, Knöpfel T, Gähwiler BH (1991) Co-cultures of inferior olive and cerebellum: electrophysiological evidence for multiple innervation of Purkinje cells by olivary axons. J Neurobiol 22:865-872.

Matsuda K, Miura E, Miyazaki T, Kakegawa W, Emi K, Narumi S, Fukazawa Y, Ito-Ishida A, Kondo T, Shigemoto R, Watanabe M, Yuzaki M (2010) Cbln1 is a ligand for an orphan glutamate receptor $\delta 2$, a bidirectional synapse organizer. Science 328:363-368.

Miyawaki A, Llopis J, Heim R, McCaffery JM, Adams JA, Ikura M, Tsien RY (1997) Fluorescent indicators for $\mathrm{Ca}^{2+}$ based on green fluorescent proteins and calmodulin. Nature 388:882-887.

Nakayama H, Miyazaki T, Kitamura K, Hashimoto K, Yanagawa Y, Obata K, Sakimura K, Watanabe M, Kano M (2012) GABAergic inhibition regu- lates developmental synapse elimination in the cerebellum. Neuron 74:384-396.

Niwa H, Yamamura K, Miyazaki J (1991) Efficient selection for highexpression transfectants with a novel eukaryotic vector. Gene 108:193-199.

Offermanns S, Hashimoto K, Watanabe M, Sun W, Kurihara H, Thompson RF, Inoue Y, Kano M, Simon MI (1997) Impaired motor coordination and persistent multiple climbing fiber innervation of cerebellar Purkinje cells in mice lacking G $\alpha$ q. Proc Natl Acad Sci U S A 94:14089-14094.

Ohno T, Sakurai M (2005) Critical period for activity-dependent elimination of corticospinal synapses in vitro. Neuroscience 132:917-922.

Paradis S, Harrar DB, Lin Y, Koon AC, Hauser JL, Griffith EC, Zhu L, Brass LF, Chen C, Greenberg ME (2007) An RNAi-based approach identifies molecules required for glutamatergic and GABAergic synapse development. Neuron 53:217-232.

Pekhletski R, Gerlai R, Overstreet LS, Huang XP, Agopyan N, Slater NT, Abramow-Newerly W, Roder JC, Hampson DR (1996) Impaired cerebellar synaptic plasticity and motor performance in mice lacking the mGluR4 subtype of metabotropic glutamate receptor. J Neurosci 16:6364-6373.

Prange O, Wong TP, Gerrow K, Wang YT, El-Husseini A (2004) A balance between excitatory and inhibitory synapses is controlled by PSD-95 and neuroligin. Proc Natl Acad Sci U S A 101:13915-13920.

Rabacchi S, Bailly Y, Delhaye-Bouchaud N, Mariani J (1992) Involvement of the $\mathrm{N}$-methyl D-aspartate (NMDA) receptor in synapse elimination during cerebellar development. Science 256:1823-1825.

Sawada Y, Kajiwara G, Iizuka A, Takayama K, Shuvaev AN, Koyama C, Hirai H (2010) High transgene expression by lentiviral vectors causes maldevelopment of Purkinje cells in vivo. Cerebellum 9:291-302.

Scheiffele P, Fan J, Choih J, Fetter R, Serafini T (2000) Neuroligin expressed in nonneuronal cells triggers presynaptic development in contacting axons. Cell 101:657-669.

Stoppini L, Buchs PA, Muller D (1991) A simple method for organotypic cultures of nervous tissue. J Neurosci Methods 37:173-182.

Sugihara I (2005) Microzonal projection and climbing fiber remodeling in single olivocerebellar axons of newborn rats at postnatal days 4-7. J Comp Neurol 487:93-106.

Tanaka M, Tomita A, Yoshida S, Yano M, Shimizu H (1994) Observation of the highly organized development of granule cells in rat cerebellar organotypic cultures. Brain Res 641:319-327.

Torashima T, Okoyama S, Nishizaki T, Hirai H (2006) In vivo transduction of murine cerebellar Purkinje cells by HIV-derived lentiviral vectors. Brain Res 1082:11-22.

Uemura T, Lee SJ, Yasumura M, Takeuchi T, Yoshida T, Ra M, Taguchi R, Sakimura K, Mishina M (2010) Trans-synaptic interaction of GluR $\delta 2$ and Neurexin through Cbln1 mediates synapse formation in the cerebellum. Cell 141:1068-1079.

Uesaka N, Hayano Y, Yamada A, Yamamoto N (2007) Interplay between laminar specificity and activity-dependent mechanisms of thalamocortical axon branching. J Neurosci 27:5215-5223.

Uesaka N, Nishiwaki M, Yamamoto N (2008) Single cell electroporation method for axon tracing in cultured slices. Dev Growth Differ 50:475-477.

Umemori H, Linhoff MW, Ornitz DM, Sanes JR (2004) FGF22 and its close relatives are presynaptic organizing molecules in the mammalian brain. Cell 118:257-270.

Varoqueaux F, Aramuni G, Rawson RL, Mohrmann R, Missler M, Gottmann K, Zhang W, Südhof TC, Brose N (2006) Neuroligins determine synapse maturation and function. Neuron 51:741-754.

Watanabe M, Kano M (2011) Climbing fiber synapse elimination in cerebellar Purkinje cells. Eur J Neurosci 34:1781-1794

Yamada A, Uesaka N, Hayano Y, Tabata T, Kano M, Yamamoto N (2010) Role of pre- and postsynaptic activity in thalamocortical axon branching. Proc Natl Acad Sci U S A 107:7562-7567.

Yamamoto N, Kurotani T, Toyama K (1989) Neural connections between the lateral geniculate nucleus and visual cortex in vitro. Science 245:192-194.

Yamasaki M, Miyazaki T, Azechi H, Abe M, Natsume R, Hagiwara T, Aiba A, Mishina M, Sakimura K, Watanabe M (2011) Glutamate receptor $\delta 2$ is essential for input pathway-dependent regulation of synaptic AMPAR contents in cerebellar Purkinje cells. J Neurosci 31:3362-3374.

Zhang LI, Poo MM (2001) Electrical activity and development of neural circuits. Nat Neurosci 4 [Suppl]:1207-1214. 